\title{
Interstellar magnetic fields in the Galactic center region
}

\author{
K. Ferrière \\ LATT, Université de Toulouse, CNRS, 14 avenue Edouard Belin, 31400 Toulouse, France \\ e-mail: ferriere@ast.obs-mip.fr
}

Received 2 June 2009 / Accepted 30 July 2009

\section{ABSTRACT}

\begin{abstract}
Aims. We seek to obtain a picture of the interstellar magnetic field in the Galactic center region that is as clear and complete as possible.

Methods. We review the observational knowledge that has built up over the past 25 years on interstellar magnetic fields within $\sim 200 \mathrm{pc}$ of the Galactic center. We then critically discuss the various theoretical interpretations and scenarios proposed to explain the existing observations. We also study the possible connections with the general Galactic magnetic field and describe the observational situation in external galaxies.

Results. We propose a coherent picture of the magnetic field near the Galactic center, which reconciles some of the seemingly divergent views and which best accounts for the vast body of observations. Our main conclusions are the following. In the diffuse intercloud medium, the large-scale magnetic field is approximately poloidal and its value is generally close to equipartition with cosmic rays $(\sim 10 \mu \mathrm{G})$, except in localized filaments where the field strength can reach $\sim 1 \mathrm{mG}$. In dense interstellar clouds, the magnetic field is approximately horizontal and its value is typically $\sim 1 \mathrm{mG}$.
\end{abstract}

Key words. ISM: magnetic fields - ISM: general - ISM: structure - cosmic rays - Galaxy: center - galaxies: magnetic fields

\section{Introduction}

The Galactic center (GC) region constitutes a very special environment, which differs from the rest of the Galaxy both by its stellar population and by its interstellar medium (ISM). Here, we are not directly concerned with the stellar population, which we tackle only to the extent that it affects the ISM, but we are primarily interested in the ISM under its various facets. More specifically, our purpose is to develop a comprehensive model of the ISM in the GC region, which can be conveniently used for a broad range of applications. The particular application we personally have in mind is to study the propagation and annihilation of interstellar positrons, which, according to the measured annihilation emission, tend to concentrate toward the central parts of the Galaxy (Knödlseder et al. 2005; Weidenspointner et al. 2006).

In a previous paper (Ferrière et al. 2007, hereafter Paper I), we focused on the interstellar gas in the Galactic bulge (GB), which we defined as the region of our Galaxy interior to a Galactocentric radius $\simeq 3 \mathrm{kpc}$. We first reviewed the current observational knowledge of its complex spatial distribution and physical state. We then used the relevant observational information in conjunction with theoretical predictions on gas dynamics near the GC to construct a parameterized model for the spaceaveraged densities of the different (molecular, atomic and ionized) gas components.

In the present paper, we direct our attention to interstellar magnetic fields. We start by providing a critical overview of some 25 years of observational and interpretative work on their properties. Since different investigation methods lead to different and sometimes contradictory conclusions, we explore the possible sources of divergence and try to filter out the dubious observational findings and the questionable theoretical interpretations. In the light of the most recent studies, we then strive to piece everything together into a coherent picture of interstellar magnetic fields in the GC region.

In Sect. 2, we give a brief summary of the main results of Paper I. In Sect. 3, we review the observational evidence on interstellar magnetic fields in the GC region, based on four different diagnostic tools. In Sect. 4, we provide a critical discussion of the various observational findings and of their current theoretical interpretations. In Sect. 5, we examine if and how the interstellar magnetic field in the GC region connects with the magnetic field in the Galaxy at large and look for additional clues from external galaxies. In Sect. 6, we present our conclusions.

\section{Spatial distribution of interstellar gas}

Following Paper I, we assume that the Sun lies at a distance $r_{\odot}=$ $8.5 \mathrm{kpc}$ from the GC, we denote Galactic longitude and latitude by $l$ and $b$, respectively, and employ two distinct Galactocentric coordinate systems:

(1) the cartesian coordinates $(x, y, z)$, with $x$ measured in the Galactic plane $\left(b=0^{\circ}\right)$ along the line of sight to the Sun (positively toward the Sun), $y$ along the line of intersection between the Galactic plane and the plane of the sky (with the same sign as $l$ ) and $z$ along the vertical axis (with the same sign as $b$ );

(2) the cylindrical coordinates $(r, \theta, z)$, with $\theta$ increasing in the direction of Galactic rotation, i.e., clockwise about the $z$-axis, from $\theta=0$ along the $x$-axis (see Fig. 1).

We further denote by $r_{\perp}$ the horizontal distance from the GC projected onto the plane of the sky, which, near the GC, is numerically given by $r_{\perp} \simeq(150 \mathrm{pc})\left(|l| / 1^{\circ}\right)$.

From the point of view of the interstellar gas, the GB possesses two prominent structural elements: the central molecular 
(a)

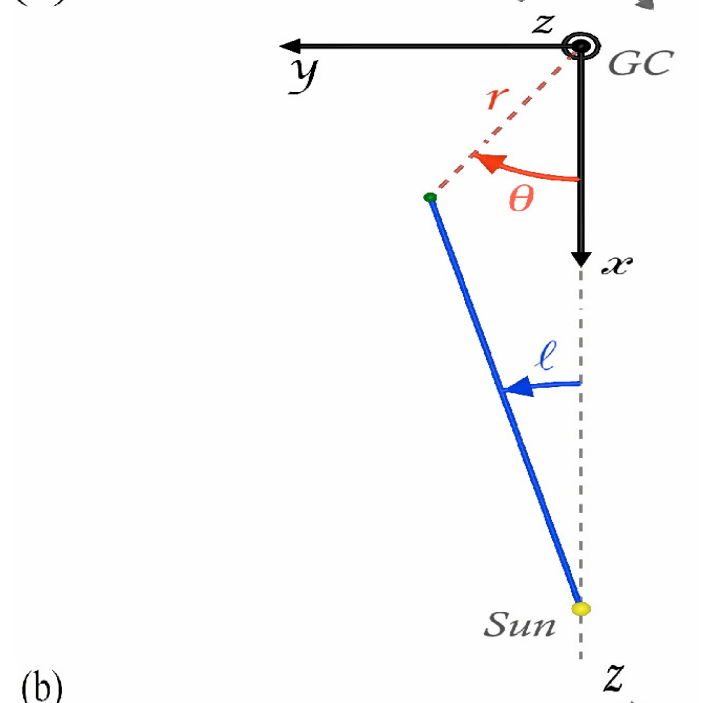

(b)

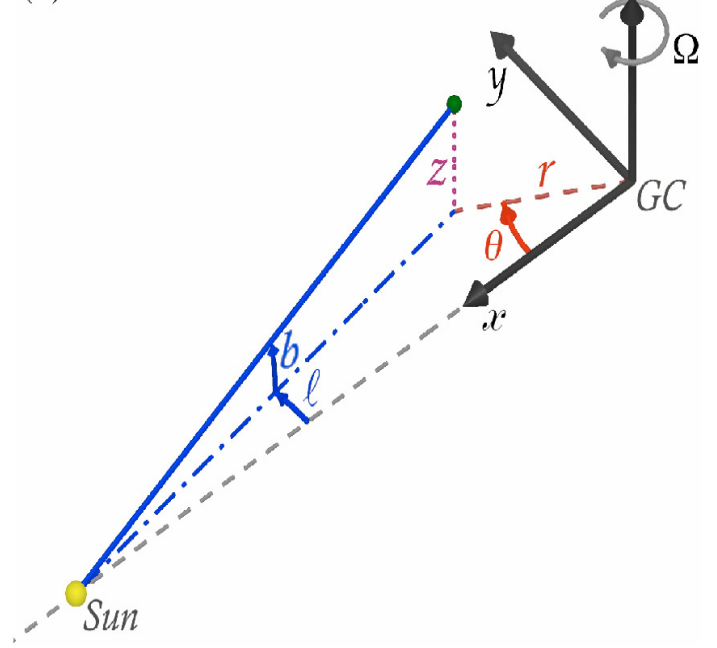

Fig. 1. Our $(x, y, z)$ and $(r, \theta, z)$ Galactocentric coordinate systems: a) face-on view from the northern Galactic hemisphere; b) full threedimensional view from a point in the fourth Galactic quadrant.

zone (CMZ) and the surrounding GB disk (also misleadingly called the HI nuclear disk) (Morris \& Serabyn 1996).

The CMZ is a thin sheet of gas, dominated at $\sim 90 \%$ by its molecular component. On the plane of the sky, it appears approximately aligned with the Galactic plane and displaced eastward by $\sim 50 \mathrm{pc}$ from the GC, it extends horizontally from $y \sim-150 \mathrm{pc}$ to $y \sim+250 \mathrm{pc}$, and it has an FWHM thickness $\sim 30 \mathrm{pc}$ in $\mathrm{H}_{2}$ and $\sim 90 \mathrm{pc}$ in HI. Its projection onto the Galactic plane was modeled in Paper I (based on the observational work of Sawada et al. 2004) as a $500 \mathrm{pc} \times 200 \mathrm{pc}$ ellipse, centered on $\left(x_{\mathrm{c}}, y_{\mathrm{c}}\right)=(-50 \mathrm{pc}, 50 \mathrm{pc})$ and inclined clockwise by $70^{\circ}$ to the line of sight. Its $\mathrm{H}_{2}$ mass was estimated at $\sim 1.9 \times 10^{7} M_{\odot}$ and its HI mass at $\sim 1.7 \times 10^{6} M_{\odot}$.

The GB disk is a much bigger structure, whose gas content is $\sim 80 \%-90 \%$ molecular. In contrast to the CMZ, it does not appear offset from the GC, but it is noticeably tilted out of the Galactic plane (counterclockwise by $\sim 7^{\circ}-13^{\circ}$ ) and inclined to the line of sight (near-side-down by as much as $\sim 20^{\circ}$ ). On the plane of the sky, it extends out to a distance $\sim 1.3 \mathrm{kpc}$ on each side of the GC, and it has an FWHM thickness $\sim 70 \mathrm{pc}$ in $\mathrm{H}_{2}$ and $\sim 200 \mathrm{pc}$ in HI. According to the model described in Paper I (itself based on the barlike model of Liszt \& Burton 1980), the
GB disk has the shape of a $3.2 \mathrm{kpc} \times 1.0 \mathrm{kpc}$ ellipse, making an angle of 51.5 clockwise to the line of sight and featuring a $1.6 \mathrm{kpc} \times 0.5 \mathrm{kpc}$ elliptical hole in the middle (just large enough to enclose the CMZ). This "holed" $\mathrm{GB}$ disk has an $\mathrm{H}_{2}$ mass $\sim 3.4 \times 10^{7} M_{\odot}$ and an $\mathrm{HI}$ mass $\sim 3.5 \times 10^{6} M_{\odot}$.

In addition to the molecular and atomic gases, which are confined either to the CMZ or to the holed GB disk, the GB also contains ionized gas, which spreads much farther out both horizontally (beyond the boundary of the GB at $r \simeq 3 \mathrm{kpc}$ ) and vertically (up to at least $|z| \simeq 1 \mathrm{kpc}$ ). This ionized gas can be found in three distinct media with completely different temperatures. The warm ionized medium (WIM), with $T \sim 10^{4} \mathrm{~K}$, is distributed into two components: an extended, $\sim 2 \mathrm{kpc}$ thick disk, which continues into the Galactic disk, and a localized, $\sim(240 \mathrm{pc})^{2} \times 40 \mathrm{pc}$ ellipsoid, nearly centered on the GC. The hot ionized medium (HIM), with $T \sim$ a few $10^{6} \mathrm{~K}$, exists throughout the entire GB. Finally, the very hot ionized medium (VHIM), with $T \gtrsim 10^{8} \mathrm{~K}$, is confined to a $\sim(270 \mathrm{pc})^{2} \times 150 \mathrm{pc}$ ellipsoid, centered on the GC and tilted clockwise by $\sim 20^{\circ}$ to the Galactic plane. In Paper I, the $\mathrm{H}^{+}$masses inside the GB were estimated at $\sim 5.9 \times 10^{7} M_{\odot}$ in the WIM (with $\sim 5.8 \times 10^{7} M_{\odot}$ in the extended disk and $\sim 6.0 \times 10^{5} M_{\odot}$ in the localized ellipsoid), $\sim 1.2 \times 10^{7} M_{\odot}$ in the HIM and $\sim 1.0 \times 10^{5} M_{\odot}$ in the VHIM.

Altogether, the interstellar hydrogen content of the GB amounts to $\sim 1.3 \times 10^{8} M_{\odot}$, with $\sim 5.3 \times 10^{7} M_{\odot}(41 \%)$ in molecular form, $\sim 5.2 \times 10^{6} M_{\odot}(4 \%)$ in atomic form and $\sim 7.1 \times 10^{7} M_{\odot}$ $(55 \%)$ in ionized form. Furthermore, if helium and metals represent, respectively, $40 \%$ and $5.3 \%$ by mass of hydrogen, the above hydrogen masses have to be multiplied by a factor 1.453 to obtain the total interstellar masses.

The broad outlines of the observed structure of the interstellar GB can be understood in terms of the theoretical properties of closed particle orbits in the gravitational potential of the Galactic bar. Basically, outside the bar's inner Lindblad resonance (ILR), particles travel along $x_{1}$ orbits, which are elongated along the bar, whereas inside the ILR, they travel along $x_{2}$ orbits, which are elongated perpendicular to the bar. In the presence of hydrodynamical (e.g., pressure and viscous) forces, the interstellar gas does not strictly follow closed particle orbits. Instead, it gradually drifts inward through a sequence of decreasing-energy orbits, and near the ILR it abruptly switches from the highenergy $x_{1}$ orbits to the lower-energy $x_{2}$ orbits. The area covered by the $x_{1}$ orbits forms a truncated disk, which can naturally be identified with our holed GB disk, and, inside the hole, the area covered by the $x_{2}$ orbits forms a smaller disk, which can be identified with the CMZ. Although the two families of particle orbits are in theory orthogonal to each other, hydrodynamical forces in the interstellar gas smooth out the $90^{\circ}$ jump in orbit orientation at the ILR, so that the CMZ actually leads the bar by less than $90^{\circ}$.

\section{Observational overview of interstellar magnetic fields}

The story begins in the 1980s, with the discovery of systems of radio continuum filaments running nearly perpendicular to the Galactic plane. The most striking of these systems is the bright radio arc (known as the Radio Arc or simply the Arc) crossing the plane near Sgr A, at $l \simeq+0.18$ (Yusef-Zadeh et al. 1984). The Radio Arc is composed of a unique set of a dozen bundled filaments, which appear long ( $\sim 40 \mathrm{pc})$, narrow $(\sim 1 \mathrm{pc})$ and remarkably regular and straight. On the other side of the GC, a fainter radio filament seems to emanate from $\operatorname{Sgr} C$, at $l \simeq-0.56$ 
(Liszt 1985). The bright Radio Arc and the fainter Sgr C filament lie vertically at the feet of the eastern and western ridges, respectively, of the $\sim 1^{\circ} \Omega$-shaped radio lobe structure observed above the GC (Sofue \& Handa 1984).

The observed morphology of the radio filaments suggests that they trace the orientation of the local interstellar magnetic field (Yusef-Zadeh et al. 1984; Morris 1990). This view has been borne out by radio polarization measurements, starting with those of Inoue et al. (1984) and Tsuboi et al. (1985, 1986 ) in the pair of polarized radio lobes (or plumes) that make up the northern and southern extensions of the Radio Arc. The authors assumed optically thin synchrotron emission and corrected the measured polarization position angles for Faraday rotation, whereupon they found that the transverse (to the line of sight) magnetic field $\mathbf{B}_{\perp}$ is indeed oriented along the axis of the plumes.

Thus, the orientation of the radio filaments provides evidence that the surrounding interstellar magnetic field is approximately normal to the Galactic plane. In addition, their slight outward curvature indicates that the magnetic field does not remain vertical at large distances from the plane, but that it turns instead to a more general poloidal geometry (Morris 1990).

Tsuboi et al. (1986) derived a crude estimate for the magnetic field strength $B$ in the polarized radio lobes, based on their measured (supposedly synchrotron) radio intensity and on the assumption of energy equipartition between the magnetic field and the energetic particles. They thereby obtained a magnetic field strength of several $10 \mu \mathrm{G}$, compatible with the line-of-sight field $\sim 10 \mu \mathrm{G}$ deduced from Faraday rotation measures (Tsuboi et al. 1985). Yusef-Zadeh \& Morris (1987b), on the other hand, estimated the equipartition field strength in the radio filaments at $\sim 0.2 \mathrm{mG}$.

Yusef-Zadeh \& Morris (1987b) also provided an independent magnetic field strength estimate for the filaments of the Radio Arc with the help of a simple dynamical argument (see also Yusef-Zadeh \& Morris 1987a). The fact that the filaments remain nearly straight all along their length and that they pass through the Galactic plane with little or no bending suggests that their magnetic pressure, $P_{\mathrm{mag}}$, is strong enough to withstand the ram pressure of the ambient interstellar clouds, $P_{\text {ram }}$. The condition $P_{\text {mag }} \gtrsim P_{\text {ram }}$, together with a conservative estimation of $P_{\text {ram }}$, then leads to the stringent requirement that $B \gtrsim 1 \mathrm{mG}$ inside the radio filaments.

A similar dynamical argument can be applied to the less prominent nonthermal radio filaments (NRFs) discovered later on. Going one step further, it is then possible to estimate the magnetic field strength outside the NRFs by invoking pressure balance. As argued by Morris (1990), the external ISM must supply a confining pressure for the NRFs. This pressure cannot be of thermal origin, because even the very hot gas, which has the highest thermal pressure, falls short by a factor $\sim 30$. The confining pressure must, therefore, be of magnetic origin, which means that the $\mathrm{mG}$ field inferred to exist inside the NRFs must also prevail outside. According to this argument, the reason why the NRFs stand out in the radio maps is not because they have a stronger magnetic field than their surroundings, but because they contain more relativistic electrons. To quote Morris (1990), the NRFs "are illuminated flux tubes within a relatively uniform field".

To sum up, the picture emerging at the end of the 1980s for the interstellar magnetic field within $\sim 70 \mathrm{pc}$ of the GC is that of a pervasive, poloidal, $\mathrm{mG}$ field. The magnetic energy contained in this $\mathrm{mG}$ field is as high as $\sim(1-2) \times 10^{54} \mathrm{erg}$ inside
$70 \mathrm{pc}$ (Morris 1990) ${ }^{1}$, which is equivalent to the energy released by $\sim(1000-2000)$ supernova explosions, and which is roughly comparable to the kinetic energy associated with Galactic rotation in the considered region, while being significantly larger than both the kinetic energy in turbulent motions and the thermal energy of the very hot gas.

The simple view of a pervasive, poloidal, $\mathrm{mG}$ field gained wide acceptance, until a variety of subsequent observations gradually called it into question. In the remainder of this section, we review the relevant observations that have either given a new twist to the simple picture described above or otherwise contributed to enhance our understanding of the interstellar magnetic field near the GC.

\subsection{Radio continuum observations}

After the initial discovery of Yusef-Zadeh et al. (1984), numerous NRFs were identified in the GC region. Although the Radio Arc is clearly a unique structure, the other NRFs share a number of observational characteristics with the Radio Arc's filaments. Morris (1996) drew up the inventory of all the GC NRFs known at the time and summarized their distinctive properties, i.e., the properties that make them unique to the GC region. In brief, the GC NRFs are a few to a few tens of parsecs long and a fraction of a parsec wide; they look straight or mildly curved along their entire length; they run roughly perpendicular to the Galactic plane; and their radio continuum emission is both linearly polarized and characterized by a spectral index consistent with synchrotron radiation.

To this list, we should add that the GC NRFs have equipartition or minimum-energy field strengths ${ }^{2}$ of several $10 \mu \mathrm{G}$, typically $\sim(50-200) \mu \mathrm{G}$ (Anantharamaiah et al. 1991; LaRosa et al. 2004 , and references therein). Since they appear to be magnetically dominated, it is likely that these values underestimate the true field strengths. This could perhaps partly explain why the equipartition/minimum-energy field strengths are systematically lower than the $\mathrm{mG}$ field strength deduced from the dynamical condition $P_{\text {mag }} \gtrsim P_{\text {ram }}$. Even so, while there is general agreement that the GC NRFs represent magnetic flux tubes lit by synchrotron-emitting electrons, the question of how strong their intrinsic magnetic field really is remains open to debate. Another unsettled question is whether their strong field is limited to their interiors or representative of the GC region as a whole.

Amongst all the NRFs cataloged by Morris (1996), the one dubbed the Snake, located at $l \simeq-0.90\left(r_{\perp} \simeq 135 \mathrm{pc}\right)$, is atypical by its morphology which exhibits two marked kinks along its length (Gray et al. 1991). This kinked shape probably reveals that the magnetic field inside the Snake is weaker than the $\mathrm{mG}$ field required to withstand the ambient ram

\footnotetext{
1 At the present time, NRFs are observed over a larger area, extending $\sim 300 \mathrm{pc}$ along the Galactic plane and $\sim 150 \mathrm{pc}$ in the vertical direction (LaRosa et al. 2004). The magnetic energy of a space-filling $\mathrm{mG}$ field in this larger region is a huge $\sim 10^{55} \mathrm{erg}$.

2 The minimum-energy state corresponds almost, but not exactly, to energy equipartition between the magnetic field and the energetic particles (Miley 1980), which, in turn, differs from pressure equipartition by a factor $\simeq 3$. Both equipartition and minimum-energy field strengths are difficult to estimate, because they depend on a number of parameters whose values are quite uncertain. In particular, they depend on the proton-to-electron energy ratio (often set to unity in this context), on the lower and upper cutoff frequencies of the synchrotron spectrum, on the spectral index, and on the line-of-sight depth of the filaments (usually taken equal to their plane-of-sky width).
} 
pressure. Incidentally, a weaker field is easier to reconcile with the minimum-energy field strength $\simeq 90 \mu \mathrm{G}$ derived by Gray et al. (1995). Since the Snake lies at a greater projected radius than the other NRFs, a simple way to explain its weaker field is to invoke an overall decrease in the interstellar magnetic field strength outside a radius $\gtrsim 100 \mathrm{pc}$.

Another interesting NRF, discovered somewhat later by Lang et al. (1999a), is the so-called Pelican, located at $l \simeq-1.15$ $\left(r_{\perp} \simeq 172 \mathrm{pc}\right)$. It, too, is noticeably kinked, but what distinguishes it most from the other NRFs is its orientation parallel to the Galactic plane. Its equipartition field strength is $\simeq 70 \mu \mathrm{G}$, and the measured polarization position angles, corrected for Faraday rotation, confirm that its intrinsic $\mathbf{B}_{\perp}$ is everywhere aligned with its long axis. The Pelican's anomalous features provide a hint that the interstellar magnetic field outside a certain radius, say, $\sim 200 \mathrm{pc}$, is not only less intense than inside $\sim 100 \mathrm{pc}$, but also oriented approximately parallel to the Galactic plane. In fact, the actual situation is probably more complex, as an NRF oriented at $45^{\circ}$ to the Galactic plane was found much closer to the GC, at $l \simeq-0.68\left(r_{\perp} \simeq 102 \mathrm{pc}\right)($ LaRosa et al. 2004).

Yusef-Zadeh et al. (2004) presented a sensitive 20-cm continuum survey of the GC region $\left(-2^{\circ}<l<5^{\circ},-40^{\prime}<b<40^{\prime}\right)$, which resulted in a new catalog of more than 80 linear radio filaments, including all the previously well-established NRFs as well as many new good candidates. The authors also drew a schematic diagram of the distribution of all the radio filaments, which puts them into perspective, with their respective positions, orientations, sizes and shapes (see their Fig. 29). A cursory look at the diagram confirms that the vast majority of filaments are nearly straight and that they have a general tendency to run along roughly vertical axes. However, upon closer inspection, it appears that only the longer filaments strictly follow this tendency. The shorter filaments exhibit a broad range of orientations, with only a loose trend toward the vertical. On the other hand, YusefZadeh et al. (2004)'s diagram does not point to any obvious correlation between either the orientation or the shape (overall curvature, presence of kinks) of the filaments and their projected distance from the GC.

The high-resolution, high-sensitivity $330 \mathrm{MHz}$ imaging survey of Nord et al. (2004) leads to similar conclusions. While the brightest NRFs (with the exception of the Pelican) tend to align along the vertical, the newly discovered fainter NRFs (or candidate NRFs) have more random orientations, with a mean angle to the vertical $\simeq 35^{\circ} \pm 40^{\circ}$. Here, too, some of the strongly inclined or nearly horizontal NRFs lie much closer to the GC than the Pelican.

The GC region was also imaged at $74 \mathrm{MHz}$ and $330 \mathrm{MHz}$ by LaRosa et al. (2005). Their high-resolution $74 \mathrm{MHz}$ image reveals, aside from discrete emission and thermal absorption features, a $6^{\circ} \times 2^{\circ}$ source of diffuse nonthermal emission centered on the GC (note that the NRFs detected at higher frequencies are resolved out in this image). The source of diffuse emission is also clearly visible in the lower-resolution $330 \mathrm{MHz}$ image. LaRosa et al. (2005) estimated the integrated $74 \mathrm{MHz}$ and $330 \mathrm{MHz}$ flux densities and the spectral index of the (supposedly synchrotron) diffuse emission, and from this they derived a minimum-energy field strength (on spatial scales $\gtrsim 5 \mathrm{pc}) \simeq(6 \mu \mathrm{G})(\phi / f)^{2 / 7}$, where $\phi$ is the proton-to-electron energy ratio and $f$ the filling factor of the synchrotron-emitting gas. They noted that even the combination of extreme values $\phi \simeq 100$ and $f \simeq 0.01$ leads to a minimum-energy field strength $\lesssim 100 \mu \mathrm{G}$. Although the large-scale magnetic field could easily be stronger than the minimum-energy value, LaRosa et al. (2005) advanced a number of cogent arguments against a large-scale field as strong as
$1 \mathrm{mG}$. With a $1 \mathrm{mG}$ field, they argued, the radiative lifetime of synchrotron-emitting electrons would only be $\sim 10^{5} \mathrm{yr}$, "shorter than any plausible replenishment timescale". In addition, the measured synchrotron flux would imply a cosmic-ray electron energy density of only $\sim 0.04 \mathrm{eV} \mathrm{cm}^{-3}$, which is about 5 times lower than in the local ISM, whereas several lines of evidence (in particular, from the observed Galactic diffuse $\gamma$-ray emission) point to comparable values.

Because of the uncertainties inherent in the equipartition/ minimum-energy assumption made to exploit radio synchrotron emission data, there have been attempts to derive the relativisticelectron density by separate means, for instance, based on Xray emission spectra. Yusef-Zadeh et al. (2005) detected X-ray emission from the radio filament G359.90-0.06, which they tentatively attributed to inverse Compton scattering of far-infrared photons from dust by the relativistic electrons that produce the radio synchrotron emission. Relying on dust thermal emission observations to constrain the dust parameters, they were able to infer the relativistic-electron density from the X-ray flux and then the magnetic field strength from the radio synchrotron flux. In this manner, they estimated the field strength in the considered NRF at $\sim(30-130) \mu \mathrm{G}$. Quite unexpectedly, this loose range of values falls below the equipartition field strength, estimated here at $\sim(140-200) \mu \mathrm{G}$.

\subsection{Faraday rotation measurements}

The first radio polarization studies of the Radio Arc by Inoue et al. (1984) and Tsuboi et al. (1985) included measurements at four different frequencies around $10 \mathrm{GHz}(\lambda=3 \mathrm{~cm})$, which enabled the authors to examine the wavelength-dependence of both the polarization position angle and the polarization degree of the incomig radiation. Inoue et al. (1984) focused on two strongly polarized regions along the Radio Arc and its extensions, which roughly coincide with the peaks in polarized intensity in the southern and northern radio lobes. They derived unusually large rotation measures $(\mathrm{RMs}) \simeq-1660 \mathrm{rad} \mathrm{m}^{-2}$ for the southern region (Source A) and $\simeq+800 \mathrm{rad} \mathrm{m}^{-2}$ for the northern region (Source B). With their assumed values of the thermal-electron density, $n_{\mathrm{e}} \sim 100 \mathrm{~cm}^{-3}$, and of the path length through the Faraday-rotating medium, $L \sim 5 \mathrm{pc}$, the derived $\mathrm{RM}$ of Source A translates into a line-of-sight magnetic field $B_{\|} \sim-4 \mu \mathrm{G}^{3,4}$ However, if Faraday rotation occurs within the synchrotron-emitting region itself (rather than in the foreground ISM), as suggested by the steep decrease in the polarization degree with increasing wavelength, then the implied value of $B_{\|}$is in fact twice higher.

Tsuboi et al. (1985), who mapped a whole $0.6 \times 1.2$ area straddling the Radio Arc, obtained positive RMs almost everywhere in the northern lobe, with a maximum value $\simeq+600 \mathrm{rad} \mathrm{m}^{-2}$ near the peak in polarized intensity, and negative RMs around the peak in polarized intensity of the southern lobe, with a minimum value $\simeq-2000 \mathrm{rad} \mathrm{m}^{-2}$ and an average value $\simeq-1500 \mathrm{rad} \mathrm{m}^{-2}$. They also found negative RMs near the northeastern edge of the northern lobe (a marginal detection, later confirmed by Tsuboi et al. (1986)) and positive RMs

\footnotetext{
3 The exact formula for the rotation measure is $\mathrm{RM}=0.81 \int n_{\mathrm{e}} B_{\|} \mathrm{d} s$,

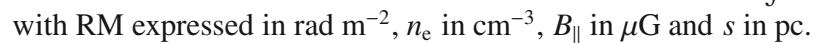

4 By convention in the Faraday rotation community, a positive (negative) value of $B_{\|}$corresponds to a magnetic field pointing toward (away from) the observer.
} 
in the extended, patchy tail of the southern lobe (not confirmed by Tsuboi et al. 1986). For the region around the peak of the southern lobe, they assumed $n_{\mathrm{e}} \sim 30 \mathrm{~cm}^{-3}$ and $L \sim 5 \mathrm{pc}$, thereby arriving at $B_{\|} \sim-10 \mu \mathrm{G}$. Again, this value should be multiplied by a factor of 2 if Faraday rotation is internal to the source of synchrotron emission.

Evidently, the line-of-sight fields inferred here from Faraday RMs can only be regarded as rough estimates, given the large uncertainties in the nature of the Faraday-rotating medium, in its location with respect to the GC (can it truly be localized to the GC?) and with respect to the synchrotron-emitting region (are both domains spatially coincident or separate?), in its lineof-sight depth, and in its thermal-electron density. But even so, the above studies bring to light a clear magnetic field reversal between the northern and southern radio lobes as well as two possible reversals near the northeastern edge of the northern lobe and between the peak and the tail of the southern lobe.

To explain the main field reversal, Tsuboi et al. (1986) invoked the presence of a magnetic flux tube running through the Galactic plane and bent somewhere near the midplane in such a way that the magnetic field globally points toward (away from) the observer above (below) the midplane. This bending could be due either to a local interaction with a nearby molecular cloud or to Galactic rotation. The secondary reversals, if confirmed, could be explained by a propagating Alfvén wave, which would also account for the waving pattern observed in the distribution of $\mathbf{B}_{\perp}$. Let us emphasize that Tsuboi et al. (1986)'s interpretation does not contradict the notion that the NRFs composing the Radio Arc are nearly straight, as the bending implied by $\left|B_{\|}\right| \sim 10 \mu \mathrm{G}$ is indeed very modest if $B \sim 1 \mathrm{mG}$. One might even see here an independent piece of evidence in support of a $\mathrm{mG}$ field, or at least, of a field $\gg 10 \mu \mathrm{G}$ - unless the bending fortuitously occurs in the vertical plane containing the line of sight.

Further, high-resolution observations at $\lambda 6 \mathrm{~cm}$ and $\lambda 20 \mathrm{~cm}$ by Yusef-Zadeh \& Morris (1987b) toward a segment of the Radio Arc comprising Source A of Inoue et al. (1984) yielded negative RMs down to $\simeq-5500 \mathrm{rad} \mathrm{m}^{-2}$ in the vicinity of Source A and positive RMs up to $\simeq+350 \mathrm{rad} \mathrm{m}^{-2}$ farther south. Similar observations by Yusef-Zadeh \& Morris (1988) toward a small area of the northern lobe comprising Source B yielded positive RMs up to $\simeq+1450 \mathrm{rad} \mathrm{m}^{-2}$. Both sets of RMs are in reasonably good agreement with the results obtained at $\lambda 3 \mathrm{~cm}$ by Inoue et al. (1984) and Tsuboi et al. (1985). However, in contrast to these authors, Yusef-Zadeh \& Morris (1988) attributed their observed RMs to a combination of internal and foreground Faraday rotation, while Yusef-Zadeh \& Morris (1987b), noticing locations with large RMs and only weak depolarization, suggested that Faraday rotation takes place outside the filaments, possibly in a helical magnetic structure surrounding them and having $L \sim 0.3 \mathrm{pc}, n_{\mathrm{e}} \sim 2200 \mathrm{~cm}^{-3}$ and $B_{\|} \sim-10 \mu \mathrm{G}$.

On the other hand, Tsuboi et al. (1995) observed the RadioArc + polarized-lobes complex at $42.5 \mathrm{GHz}(\lambda=0.7 \mathrm{~cm})$, a higher frequency at which Faraday rotation and Faraday depolarization should be nearly negligible. Not only did they confirm the RMs derived by Inoue et al. (1984) and Tsuboi et al. (1985), but they also made their case for internal Faraday rotation stronger. Clearly, the contradictory interpretations proposed by different authors for the Radio Arc and polarized lobes attest to the difficulty of locating the Faraday screen with respect to the synchrotron source.

Let us now turn to the Snake (see Sect. 3.1), which was observed by Gray et al. (1995) at five different frequencies ranging between $0.84 \mathrm{GHz}$ and $8.64 \mathrm{GHz}$. By analyzing the wavelengthdependence of its polarization properties, they found that the
Snake experiences both internal Faraday rotation, with Faraday depth $\sim+1400 \mathrm{rad} \mathrm{m}^{-2}$, and foreground Faraday rotation, with $\mathrm{RM} \sim+5500 \mathrm{rad} \mathrm{m}^{-2}$. For the external Faraday-rotating medium, they estimated $n_{\mathrm{e}} \sim 10 \mathrm{~cm}^{-3}, L \sim 100 \mathrm{pc}$ and thus $B_{\|} \sim 7 \mu \mathrm{G}$, while for the Snake itself, they mentioned that the measured internal Faraday depth could be reproduced with $n_{\mathrm{e}} \sim 10 \mathrm{~cm}^{-3}$, $L \sim 1$ pc (approximate width of the Snake) and $B_{\|} \sim 90 \mu \mathrm{G}$ (minimum-energy field strength; see Sect. 3.1). Note that the latter value is not particularly relevant, as there is no reason why $B_{\|}$ (which is probably only a minor component of $\mathbf{B}$ ) would equal the minimum-energy field strength.

Another instructive NRF is G359.54+0.18, observed by Yusef-Zadeh et al. (1997) at $\lambda 3.6 \mathrm{~cm}$ and $\lambda 6 \mathrm{~cm}$. The authors obtained RMs down to $\simeq-4200 \mathrm{rad} \mathrm{m}^{-2}$, with typical values in the range $\sim-3000 \mathrm{rad} \mathrm{m}^{-2}$ to $-1500 \mathrm{rad} \mathrm{m}^{-2}$. They argued that Faraday rotation occurs in a foreground screen, which they suggested could be the $10^{8} \mathrm{~K}$ gas present in the inner $100 \mathrm{pc}$ of the Galaxy. With $n_{\mathrm{e}} \sim 0.03 \mathrm{~cm}^{-3}$ (Yamauchi et al. 1990), this gas would require $B_{\|} \sim-1 \mathrm{mG}$ to explain the typical RM values. And if, as suggested by the small-scale RM variations along the filament, the $10^{8} \mathrm{~K}$ gas is clumped with a filling factor $\Phi \sim 0.01$, the values of $n_{\mathrm{e}}$ and $B_{\|}$should both be divided by $\sqrt{\Phi} \sim 0.1$, which would make $\left|B_{\|}\right|$even more unrealistically high. However, we note that the $10^{8} \mathrm{~K}$ gas encloses but a small fraction of the interstellar free electrons on the line of sight, the vast majority of them residing in the warm $\sim 10^{4} \mathrm{~K}$ phase of the ISM (see Paper I). Accounting for all the interstellar free electrons, supposed to be distributed according to Cordes \& Lazio (2002)'s model, we find that their integrated density from the GC to Galactic radius $r$ rises steeply with $r$ out to $\sim 200 \mathrm{pc}$, with $\int_{0}^{200 \mathrm{pc}} n_{\mathrm{e}} \mathrm{d} s \simeq 1230 \mathrm{~cm}^{-3} \mathrm{pc}$, then gradually levels off to $\int_{0}^{r_{\odot}} n_{\mathrm{e}} \mathrm{d} s \simeq 1960 \mathrm{~cm}^{-3} \mathrm{pc}$. It then follows that the above typical RMs translate into $\bar{B}_{\|} \sim-(1-2) \mu \mathrm{G}$, where the overbar denotes a line-of-sight average weighted by $n_{\mathrm{e}}$, i.e., in the present context, a line-of-sight average heavily weighted toward the region $r \lesssim 200$ pc. Obviously, $\bar{B}_{\|}$could significantly underestimate the typical local $B_{\|}$in this region if the magnetic field reverses along the line of sight.

For the Pelican, observed by Lang et al. (1999a) at $\lambda 3.6 \mathrm{~cm}$, $\lambda 6 \mathrm{~cm}$ and $\lambda 20 \mathrm{~cm}$, the measured RMs vary smoothly from $\simeq-500 \mathrm{rad} \mathrm{m}^{-2}$ at the western end to $\simeq+500 \mathrm{rad} \mathrm{m}^{-2}$ at the eastern end, with a peak value $\simeq-1000 \mathrm{rad} \mathrm{m}^{-2}$. Faraday rotation is most probably external, as implied by the particularly high polarization degrees. If so, the fact that the Pelican has somewhat lower RMs than the other NRFs could indicate that it lies slightly closer to the Sun, and the sign reversal in RM could reflect the presence of a magnetic perturbation in front of the Pelican, with field lines bent out of the plane of the sky. Otherwise, the authors made no attempt to convert their measured RMs into estimates for $B_{\|}$.

More RM studies toward other NRFs have been carried out in the last decade (e.g., Lang et al. 1999b). The derived RMs typically range from a few hundred to a few thousand $\mathrm{rad} \mathrm{m}^{-2}$. When these RMs are used to estimate $B_{\|}$near the GC, values of a few $\mu \mathrm{G}$ are usually obtained. As already mentioned above, these values are extremely uncertain, due to our poor knowledge of the precise free-electron density distribution along the considered line of sight. Furthermore, their exact significance remains unclear, first because they refer to line-of-sight averages (with possible cancellations if $B_{\|}$reverses sign) through ionized regions only, and second because $B_{\|}$is likely to represent but a small component of the total magnetic field. In this respect, the 
inferred values of $B_{\|}$might tell us more about the rigidity of the interstellar magnetic field near the GC than about its strength.

RM studies also provide valuable information on the magnetic field geometry near the GC. By collecting all the available RMs toward NRFs within $1^{\circ}$ of the GC, Novak et al. (2003) were able to uncover a definite pattern in the sign of RM, such that $\mathrm{RM}>0$ in the quadrants $(l>0, b>0)$ and $(l<0, b<0)$ and $\mathrm{RM}<0$ in the quadrants $(l>0, b<0)$ and $(l<0, b>0)$. This pattern, they explained, could be understood as the result of an initially axial magnetic field (pointing north) being sheared out in the azimuthal direction by the Galactic differential rotation (dense gas near the Galactic plane tends to rotate faster than diffuse gas higher up). The RM results described in this section show that the actual situation is not as clear-cut, as we came across several filaments that exhibit both signs of RM in the same quadrant. For the filaments of the Radio Arc, one sign clearly dominates, and the other sign could be attributed to an Alfvén wave traveling along the filaments (Tsuboi et al. 1986). For the Pelican (which lies slightly outside $1^{\circ}$ of the GC), both signs are equally important, and the sign reversal could be attributed to a foreground magnetic perturbation (see above).

Novak et al. (2003)'s conclusions are not supported by the recent work of Roy et al. (2005) and Roy et al. (2008), who considered the more extended area $\left(|l|<6^{\circ},|b|<2^{\circ}\right)$. Roy et al. (2005) observed 59 background extragalactic sources through this area, at $\lambda 3.6 \mathrm{~cm}$ and $\lambda 6 \mathrm{~cm}$, and obtained RMs in the range $\simeq-1180 \mathrm{rad} \mathrm{m}^{-2}$ to $+4770 \mathrm{rad} \mathrm{m}^{-2}$. Roy et al. (2008) remarked that these RMs are predominantly positive, with a mean value $\simeq+413 \mathrm{rad} \mathrm{m}^{-2}$, and they found no evidence for a RM sign reversal either across the rotation axis or across the midplane. As discussed by the authors, this observed RM distribution is consistent with either the large-scale Galactic magnetic field having a bisymmetric spiral configuration or the magnetic field in the central region of the Galaxy being oriented along the Galactic bar. Interestingly, the 4 extragalactic sources of the sample that fall closer than $1^{\circ}$ of the GC conform neither to Novak et al. (2003)'s pattern (only 2 have the expected RM sign) nor to the general pattern of the more extended area (only 2 have $R M>0$ ).

It is clear that a much broader sample of RM data toward the GC would be needed to draw firm conclusions on the magnetic field geometry near the GC. It would also be necessary to get a better handle on how much of the observed RMs can truly be attributed to the GC region.

Roy et al. (2008) argued that their RMs have negligible contributions from the sources themselves, they estimated the contribution from the Galactic disk to be small and, based on the measured RMs of 7 pulsars located within the survey area (with distances between $1.5 \mathrm{kpc}$ and $7.7 \mathrm{kpc}$ and with a mean $\mathrm{RM}$ of only $\left.\simeq(-7 \pm 46) \mathrm{rad} \mathrm{m}^{-2}\right)$, they ruled out the possibility that a single high-RM object (HII region or supernova remnant) could bias their entire sample. From this, they concluded that their RMs arise mainly from the central $\sim 2 \mathrm{kpc}$ of the Galaxy. Relying on Bower et al. (2001)'s observations, they further estimated the free-electron column density across the central $2 \mathrm{kpc}$ at $\sim 800 \mathrm{~cm}^{-3} \mathrm{pc}$, which they combined with the mean RM $\simeq+413 \mathrm{rad} \mathrm{m}^{-2}$ to derive a mean $\bar{B}_{\|} \sim 0.6 \mu \mathrm{G}$. Again, this value by itself is not very meaningful, as it represents an $n_{\mathrm{e}}$-weighted average, over a $12^{\circ} \times 4^{\circ}$ cone opening up from the Sun toward the $\mathrm{GC}$, of a quantity that undergoes repeated sign reversals. More useful is the estimate of the turbulent (or random) component of $B_{\|}$. From the RM structure function and the r.m.s RM of their sample, Roy et al. (2008) estimated $\delta B_{\|} \sim 6 \mu \mathrm{G}$ in the region $r \lesssim 1 \mathrm{kpc}$ and $\delta B_{\|} \sim 20 \mu \mathrm{G}$ in the region $r \lesssim 150 \mathrm{pc}$.

\subsection{Infrared and sub-millimeter polarimetry}

Interstellar dust grains tend to spin about their short axes and to align the latter along the local magnetic field. As a result, the dust thermal emission, at far-infrared (FIR) and sub-millimeter (Smm) wavelengths, is linearly polarized perpendicular to the ambient magnetic field. It then follows that FIR/Smm polarimetry makes it possible to map out the direction of the interstellar magnetic field on the plane of the sky, in regions of strong dust emissivity, i.e., in high-density regions (e.g., Hildebrand 1988). The first successful applications of this technique to the GC area were by Aitken et al. (1986, 1991).

Davidson (1996) reviewed the existing FIR polarization measurements toward dense regions located within $\sim 100 \mathrm{pc}$ of the GC. In the four regions that she discussed (certainly in the Circumnuclear Disk ${ }^{5}$ (CND) and associated Northern Streamer, in the Arched Filaments and in the Sickle, and possibly in Sgr B2), the measured field direction is roughly parallel to the Galactic plane. Davidson (1996) argued that this field direction could be explained by the dense gas moving relative to the surrounding diffuse gas and either distorting the local poloidal magnetic field or dragging its own distorted field from another Galactic location. She also quoted a mean magnetic field strength $\sim 6 \mathrm{mG}$ in the Arched Filaments, as inferred from the Chandrasekhar-Fermi relation (see Morris et al. 1992).

Compared to FIR polarimetry, which probes the warmer parts of molecular clouds, Smm polarimetry applies to their colder parts. Polarized Smm emission from the GC region was first detected by Novak et al. (2000), who carried out $350 \mu \mathrm{m}$ polarimetric observations of three separate $2^{\prime} \times 2^{\prime}$ areas, centered on the CND and on the peaks of the M-0.02-0.07 and M-0.13-0.08 molecular clouds, respectively. In all three areas, the measured field directions are more-or-less inclined to the Galactic plane. In the CND, they are similar to the field directions given by FIR polarimetry; in the curved ridge of $\mathrm{M}-0.02-0.07$, they are everywhere aligned with the ridge, consistent with the field being compressed together with the gas by the expansion of Sgr A East; and in M-0.13-0.08, they are on average parallel to the cloud long axis, consistent with the field being stretched out by the tidal forces that gave the cloud its elongated shape. These first results already show that the magnetic field configuration near the GC is governed by a combination of different factors.

A much larger area about the GC, extending $170 \mathrm{pc}$ in longitude and $30 \mathrm{pc}$ in latitude, was observed in $450 \mu \mathrm{m}$ polarized emission by Novak et al. (2003). Their polarization map clearly shows that the magnetic field threading molecular clouds is, on the whole, approximately parallel to the Galactic plane. To reconcile the horizontal field measured in molecular clouds with the poloidal field traced by NRFs, Novak et al. (2003) suggested that the large-scale magnetic field near the GC is predominantly poloidal in the diffuse ISM and predominantly toroidal in dense regions along the Galactic plane, where it was sheared out in the azimuthal direction by the differential rotation of the dense gas.

Chuss et al. (2003) reported further $350 \mu \mathrm{m}$ polarimetric observations of the central $50 \mathrm{pc}$ of the Galaxy, which led them to refine the conclusions of Novak et al. (2003). They found that the measured field direction depends on the molecular gas

\footnotetext{
5 The Circumnuclear Disk is a compact torus of neutral (mainly molecular) gas and dust surrounding Sgr A West (the HII region centered on the point-like source Sgr $\mathrm{A}^{*}$ ) and extending between $\sim 1.5 \mathrm{pc}$ and $7 \mathrm{pc}$ from the GC (Morris \& Serabyn 1996; Mezger et al. 1996).
} 
density in such a way that it is generally parallel to the Galactic plane in high-density regions and generally perpendicular to it in low-density regions. They proposed two possible scenarios to explain their results. In their preferred scenario, the largescale magnetic field was initially poloidal everywhere, but in dense molecular clouds, where the gravitational energy density exceeds the magnetic energy density, it became sheared out into a toroidal field by the clouds' motions. In the alternative, less likely scenario, the large-scale magnetic field was initially toroidal everywhere, but outside dense molecular clouds, it became distorted into a poloidal field by winds due to supernova explosions. If the first scenario is correct, a characteristic field strength inside GC molecular clouds can be estimated by assuming that clouds where the field is half-way between toroidal and poloidal (i.e., inclined by $45^{\circ}$ to the vertical) are those where gravitational and magnetic energy densities are equal. A crude estimation of the gravitational energy then yields a characteristic field strength $\sim 3 \mathrm{mG}$ inside molecular clouds ${ }^{6}$

While dust grains emit polarized thermal radiation at FIR/Smm wavelengths, they absorb starlight at optical and nearinfrared (NIR) wavelengths. Optical starlight from the GC region becomes completely extinct before reaching us, but NIR starlight suffers only partial extinction, such that it reaches us linearly polarized in the direction of the magnetic field (the dustweighted average field along the line of sight to the observed stars). For this reason, NIR polarimetry toward the GC has become a new tool to trace the average magnetic field direction on the plane of the sky, in the GC region.

Nishiyama et al. (2009) obtained a NIR polarization map of a $50 \mathrm{pc} \times 50 \mathrm{pc}$ area centered on the GC. Compared to earlier NIR polarimetric observations toward the GC (e.g., Eckart et al. 1995; Ott et al. 1999), they were able, for the first time, to separate out the contribution from foreground dust and to isolate the polarization arising within $\sim(1-2) \mathrm{kpc}$ of the GC. Their inferred distribution of polarization position angles exhibits a strong peak in a direction nearly parallel to the Galactic plane, in good agreement with the results of FIR/Smm polarimetry. However, in contrast to Chuss et al. (2003), Nishiyama et al. (2009) found no indication that the magnetic field direction depends on gas density - the field appears to be everywhere horizontal, including in the diffuse ISM.

These first NIR results are preliminary, but they demonstrate the potential of NIR polarimetry to probe the GC magnetic field. This potential will probably prove particularly valuable in directions where the FIR/Smm emission flux is too weak to perform FIR/Smm polarimetry.

\subsection{Zeeman splitting measurements}

The line-of-sight magnetic field in dense, neutral (atomic or molecular) regions can in principle be measured directly through Zeeman splitting of radio spectral lines. In practice, though, near the GC, the task is made difficult by the very broad linewidths of GC clouds and by the line-of-sight blending of physically unrelated spectral features. The first factor sets a stringent

\footnotetext{
6 This rough estimate of $B$ was necessarily going to be larger than the $\mathrm{mG}$ estimate derived for the diffuse ISM by Yusef-Zadeh \& Morris (1987a,b) from the dynamical condition $P_{\text {mag }} \gtrsim P_{\text {ram }}$ (see beginning of Sect. 3), since the orbital velocity of molecular clouds in the Galactic gravitational potential $\left(\sim 150 \mathrm{~km} \mathrm{~s}^{-1}\right)$ exceeds their turbulent velocity $\left(\sim 15 \mathrm{~km} \mathrm{~s}^{-1}\right)$.
}

observational limit ( $B_{\|} \sim$ a few $0.1 \mathrm{mG}$ ), below which magnetic fields cannot be detected with this method.

The first Zeeman splitting measurements toward the GC date back to the early 1990s and pertain to the CND. Schwarz \& Lasenby (1990) measured the Zeeman splitting of the HI 21-cm absorption line and reported the tentative detection of $B_{\|} \sim+0.5 \mathrm{mG}^{7}$ near the northern edge of the CND as well as a $3 \sigma$ upper limit $\sim 1.5 \mathrm{mG}$ near the southern edge. From the Zeeman splitting of the $\mathrm{OH} 1667-\mathrm{MHz}$ absorption line, Killeen et al. (1992) derived $B_{\|} \sim-2 \mathrm{mG}$ both in the southern part (firm detection) and in the northern part (marginal detection) of the $\mathrm{CND}$; for other $\mathrm{OH}$ clouds within the central $\sim 200 \mathrm{pc}$, they obtained $3 \sigma$ upper limits $\sim(1-2) \mathrm{mG}$. Marshall et al. (1995) and Plante et al. (1995) searched further for Zeeman splitting in HI 21-cm absorption over different areas of the CND. While the former found only an upper limit $\sim 0.5 \mathrm{mG}$ for each of the northern and southern parts of the CND, the latter reported 7 detections ( 1 positive and 6 negative values of $B_{\|}$) ranging between $-4.7 \mathrm{mG}$ and $+1.9 \mathrm{mG}$ toward the northern part of the CND; the 6 strongest detections were argued to arise from the Northern Streamer rather than from the CND itself.

The disparate Zeeman results obtained for the CND are not necessarily contradictory; they can be reconciled if $B_{\|}$varies substantially - especially if $B_{\|}$changes sign - across the CND. In that case, averaging over broad portions of the CND lowers the overall Zeeman signal, and sometimes does so to below the detectability threshold (Marshall et al. 1995; Plante et al. 1995).

Zeeman splitting measurements outside the CND were performed by Uchida \& Guesten (1995), who observed 13 selected positions within a few degrees of the $\mathrm{GC}$, in the $\mathrm{OH} 1665-\mathrm{MHz}$ and $1667-\mathrm{MHz}$ absorption lines. The large velocities and broad linewidths of the absorption features together with the relatively high molecular densities required to produce the observed absorption made the authors confident that the absorbing clouds are located close to the GC. All the measurements led to nondetections, with $3 \sigma$ upper limits to $B_{\|} \sim(0.1-1) \mathrm{mG}$. Here, beam dilution of the Zeeman signal, added to line-of-sight averaging, could be partly responsible for the absence of detections. Nonetheless, Uchida \& Guesten (1995)'s negative results provide evidence that the mean (or uniform) magnetic field through GC molecular clouds is either weaker than the $\mathrm{mG}$ field believed to thread NRFs or nearly perpendicular to the line of sight. The latter possibility seems ruled out for some of the considered clouds, notably, for the cloud associated with the Arched Filaments, whose observed line-of-sight-velocity gradient should be accompanied by field-line shearing along the line of sight. More generally, FIR/Smm polarimetry indicates that the magnetic field inside molecular clouds is approximately horizontal (see Sect. 3.3), which makes it unlikely that all the clouds studied by Uchida \& Guesten (1995) would have their magnetic field nearly perpendicular to the line of sight. From this, one might conclude that the mean magnetic field through GC molecular clouds is generally below the $\mathrm{mG}$ level.

On the other hand, Crutcher et al. (1996) mapped the Zeeman effect in HI 21-cm absorption toward the Main and North cores of Sgr B2, and they reported values of $B_{\|}$at 4 different locations, all between $\simeq-0.1 \mathrm{mG}$ and $-0.8 \mathrm{mG}$. Sgr B2 corresponds

\footnotetext{
7 The convention for the sign of $B_{\|}$in the Zeeman splitting community is opposite to that adopted in the Faraday rotation community. Here, a positive (negative) value of $B_{\|}$corresponds to a magnetic field pointing away from (toward) the observer. Note that in their original paper, Schwarz \& Lasenby (1990) mistakenly quoted a negative $B_{\|}$; the error was later corrected by Killeen et al. (1992).
} 
to one of the positions examined by Uchida \& Guesten (1995), for which they managed to derive neither a value of $B_{\|}$nor an upper limit to $B_{\|}$. This failure to obtain information on $B_{\|}$was due to a combination of coarse angular resolution $\left(\sim 8^{\prime}\right.$, as opposed to $\sim 10^{\prime \prime}$ for Crutcher et al. (1996)'s map), small-scale magneticfield structure and blending of the $\mathrm{OH}$ absorption lines with $\mathrm{OH}$ maser emission lines.

Zeeman splitting of $\mathrm{OH}(1720 \mathrm{MHz})$ maser emission from the Sgr A region was measured by Yusef-Zadeh et al. (1996, 1999). The preliminary analysis of Yusef-Zadeh et al. (1996) yielded very strong magnetic fields, with $B_{\|} \sim+(2.0-3.7) \mathrm{mG}$ in the Sgr A East shell and $B_{\|} \sim-(3.0-4.0) \mathrm{mG}$ in the CND. Follow-up, higher-resolution observations by Yusef-Zadeh et al. (1999) confirmed the presence of very strong fields, with $B_{\|}$ reaching $\sim+3.7 \mathrm{mG}$ in Sgr A East and $\sim-4.8 \mathrm{mG}$ in the CND. One should, however, keep in mind that $\mathrm{OH}$ masers arise in very special environments, for instance, in highly compressed regions behind interstellar shock waves. Therefore, field strengths inferred from $\mathrm{OH}$ maser emission lines may a priori not be considered typical of the ISM near the GC.

Like in Faraday rotation studies, Zeeman splitting results have to be taken with caution. When a true detection is made, it provides only a lower limit to the local $B_{\|}$in the observed region, insofar as the Zeeman signal is reduced (sometimes severely) by averaging over the observed area and along the line of sight. In addition, $B_{\|}$itself represents only a fraction of the total field strength, though not necessarily a small fraction as in Faraday rotation studies, given that the magnetic field in dense regions is probably nearly horizontal (see Sect. 3.3). It should also be emphasized that the magnetic field in dense regions might not be representative of the magnetic field in the general ISM: like in the Galactic disk, molecular clouds near the GC are likely to harbor much stronger fields than their surroundings.

\section{Discussion}

A fragmentary picture of the interstellar magnetic field in the $\mathrm{GC}$ region (over $\sim 300 \mathrm{pc}$ along the Galactic plane and $\sim 150 \mathrm{pc}$ in the vertical direction) emerges from the overview given in Sect. 3. In the diffuse intercloud medium, sampled by the observed NRFs, the field appears to be approximately poloidal on average, with considerable scatter (see Sect. 3.1), whereas in dense interstellar clouds, probed by FIR/Smm polarimetry, the field appears to be approximately horizontal (see Sect. 3.3). The field strength, $B$, is still a matter of controversy: in NRFs, $B$ lies somewhere between $\sim 100 \mu \mathrm{G}$ (equipartition/minimum-energy field strength, supported by inverse Compton scattering data) and $\gtrsim 1 \mathrm{mG}$ (dynamical condition $P_{\text {mag }} \gtrsim P_{\text {ram }}$ ); in the general intercloud medium, $B$ lies between $\sim 10 \mu \mathrm{G}$ (minimum-energy field strength) and $\sim 1 \mathrm{mG}$ (assumption of pressure balance with the NRFs); and for dense interstellar clouds, some Zeeman splitting measurements yield $\left|B_{\|}\right| \sim(0.1-1) \mathrm{mG}$ (with values up to a few $\mathrm{mG}$ in the Sgr A region), while others lead only to upper limits $\sim(0.1-1) \mathrm{mG}$.

\subsection{Magnetic field direction: theoretical interpretation}

The approximately poloidal direction of the large-scale magnetic field in the intercloud medium near the GC can be explained in different ways. The simplest explanation, originally proposed by Sofue \& Fujimoto (1987), is that the inflow of Galactic disk matter that presumably created the $\mathrm{CMZ}$ dragged along the primordial Galactic magnetic field and compressed its vertical component into the CMZ, while letting its horizontal component diffuse away perpendicular to the disk. This idea was elaborated upon by Chandran et al. (2000), who emphasized the importance of vertical ambipolar diffusion in removing horizontal magnetic flux, especially in the CMZ where the vertical ambipolar diffusion velocity (which is proportional to $B^{2}$ ) reaches particularly high values. Neglecting outward turbulent diffusion, Chandran et al. (2000) estimated that a pregalactic vertical field $\sim 0.2 \mu \mathrm{G}$ was required to account for the present-day CMZ field, which they supposed to be $\sim 1 \mathrm{mG}$.

A pregalactic magnetic field as strong as $0.2 \mu \mathrm{G}$ might be unrealistic, unless field amplification occurred during the very process of galaxy formation - for instance, through turbulent dynamo action in the protogalaxy (Kulsrud et al. 1997; Kulsrud \& Zweibel 2008). But even if the pregalactic field was weaker, the scenario of inward field advection might still be adequate provided that the large-scale vertical field was rapidly amplified (to nearly its present-day value) by a dynamo operating in the Galactic disk and that its direction remained constant both in time and in space (at least out to $\sim 10 \mathrm{kpc}$ from the GC). To show this quantitatively, we now derive a rough upper limit to the CMZ field strength that might be expected under these conditions. At the Galactic position of the Sun $\left(r_{\odot}=8.5 \mathrm{kpc}\right)$, the present-day large-scale vertical field can be inferred from Faraday rotation measures of high-latitude Galactic pulsars and extragalactic radio sources; Han et al. (1999) thus derived $B_{z} \simeq$ $0.37 \mu \mathrm{G}$, while Sui et al. (2009) recently obtained $B_{z} \simeq 0 \mu \mathrm{G}$ toward the North Galactic Pole and $B_{z} \simeq 0.5 \mu \mathrm{G}$ toward the South Galactic Pole. Here, we adopt $B_{z} \simeq 0.25 \mu \mathrm{G}$ as a conservative estimate. We further assume that the interstellar plasma, together with the frozen-in field lines, slowly drift inward, with a radial velocity at the solar circle $v_{r} \simeq-(0.5-1.0) \mathrm{km} \mathrm{s}^{-1}$ (taken from the Galactic evolution models of Lacey \& Fall 1985). If these values apply over most of the $\sim 10^{10}$ yr lifetime of the Galaxy and if the inflowing vertical magnetic flux accumulates inside the $\mathrm{CMZ}$ at roughly the same rate as it crosses the solar circle, then the resulting present-day vertical field in the CMZ (whose projection onto the Galactic plane can be approximated as a 500 pc $\times 200$ pc ellipse; see Sect. 2$)$ is $\sim(0.85-1.7) \mathrm{mG}$. This estimate is in surprisingly close agreement with the $\sim 1 \mathrm{mG}$ observational value assumed by Chandran et al. (2000). However, the agreement may be a little fortuitous, and one has to bear in mind that our estimate is contingent upon a number of important and uncertain hypotheses, such as rapid dynamo amplification in the Galactic disk, no sign reversals in the large-scale vertical field, steady-state inflow and accumulation into the CMZ, negligible turbulent diffusion out of the CMZ... If one or more of these hypotheses fail, the actual CMZ field is less than estimated here.

Another category of scenarios rely on outflows from the Galactic nucleus. In one such scenario, a magnetic field was generated in the accretion disk around the central black hole, through creation of a seed field by a Biermann battery and subsequent amplification of this seed field either by the large-scale shear alone or by a standard dynamo; the generated field was then expelled into the surrounding ISM by Galactic winds or collimated outflows (Chakrabarti et al. 1994) ${ }^{8}$. A priori, the strong shear in the accretion disk must have caused the generated field

8 In a related model (Heyvaerts et al. 1988), the central accretion disk generates magnetic loops which break off and expand away from their source. Although this model provides a possible explanation for the 
to be nearly toroidal. Therefore, if the accretion disk was parallel to the Galactic plane and if the field was expelled horizontally, this process alone could be a good candidate to explain a horizontal large-scale magnetic field in the GC region, but not a poloidal field. This horizontal field would only be a few $\mu \mathrm{G}$, unless, for some reason, the expelled field remained confined to the CMZ. In this case, the horizontal large-scale magnetic field in the CMZ (approximated again as a $500 \mathrm{pc} \times 200 \mathrm{pc}$ ellipse) would be $\sim(0.25-0.6) \mathrm{mG}$, as can be found by using the expression of the nuclear field provided by Chakrabarti et al. (1994), together with a central black hole mass $\simeq 4 \times 10^{6} M_{\odot}$ (Gillessen et al. 2009; Ghez et al. 2008). In reality, magnetic activity in the immediate vicinity of the central black hole is certainly more complex than described above. As it turns out, recent NIR polarimetric imaging observations of Sgr $\mathrm{A}^{*}$, in conjunction with earlier radio, infrared and X-ray data, provide loose evidence that the accretion disk might indeed be nearly parallel to the Galactic plane (Trippe et al. 2007; Meyer et al. 2007, and references therein). However, they also suggest that the nuclear field switches back and forth between toroidal (as expected from strong shear) and poloidal (consistent with recurrent vanishing of the accretion disk). And even if the nuclear field were strictly toroidal, it could, after ejection, be twisted by the Coriolis force into a more poloidal field.

Still in the category of outflow scenarios, a poloidal largescale magnetic field in the GC region could find its roots in stellar activity very close to the GC (e.g., Sofue 1984; Chevalier 1992) - for instance, in a relatively recent nuclear starburst (e.g., Bland-Hawthorn \& Cohen 2003). To start with, stellar fields are continuously injected into the ISM via stellar winds and supernova explosions; stellar fields alone cannot account for the present-day interstellar magnetic field, but one could conceive that a very early generation of them provided the seed field for a local dynamo (see below). More relevant here, the energy release at the GC must have driven the interstellar matter into expanding motions and created a vertically elongated shell structure, or wall, into which interstellar field lines were pushed back and compressed. This mechanism was first invoked by Sofue (1984) to explain the $\Omega$-shaped radio lobe detected by Sofue \& Handa (1984), and it was further studied by Lesch et al. (1989) and by Chevalier (1992). The result is that a pre-existing horizontal field could have turned into a seemingly poloidal configuration (as seen from the Sun), while a pre-existing vertical field could have been substantially enhanced at the position of the shell.

Finally, with the strong shear flows and the highly turbulent motions existing near the GC, dynamo action there is almost inescapable. Chandran et al. (2000) argued that dynamo amplification in the CMZ is unlikely, on the grounds that the magnetic field there is way above equipartition with the turbulence. However, their argument is based on a supposed $\mathrm{mG}$ field, which we now regard as a probable overestimate (see Sect. 4.2). If we assume that the $\mathrm{CMZ}$ is characterized by a turbulent velocity $v_{\text {turb }} \sim 15 \mathrm{~km} \mathrm{~s}^{-1}$ (value adopted by Yusef-Zadeh \& Morris (1987a,b) for the cloud random velocity), a space-averaged hydrogen density $\left\langle n_{\mathrm{H}}\right\rangle \sim 300 \mathrm{~cm}^{-3}$ (see Paper I) and a total-tohydrogen mass ratio of 1.453 (see Sect. 2), we find that turbulent pressure corresponds to the magnetic pressure of $0.2 \mathrm{mG}$ field. In consequence, the saturated field strength expected from dynamo action in the CMZ is $\sim 0.2 \mathrm{mG}$, and any field $\lesssim 0.2 \mathrm{mG}$ would be dynamically consistent with dynamo amplification in the $\mathrm{CMZ}$

NRF phenomenon, it does not discuss the origin of the large-scale magnetic field in the $\mathrm{CG}$ region.
- or, more generally, in a region encompassing the CMZ and the ionized gas around it.

If the large-scale magnetic field in the GC region was indeed amplified by a local dynamo, its final configuration must depend on the shape of the dynamo domain. Moss \& Sokoloff (2008) showed that in astrophysical objects composed of a flattened disk-like structure and a quasi-spherical halo-like structure (such as spiral galaxies), a dynamo generates a quadrupolar (i.e., symmetric in $z$ ) field if the disk-like structure is more dynamo-active and a dipolar (i.e., anti-symmetric in $z$ ) field if the halo-like structure is more dynamo-active. This theoretical result applied to the $\mathrm{GC}$ region implies that a dynamo field there must be quadrupolar if the disk-like CMZ dominates dynamo action and dipolar if the surrounding halo-like volume of ionized gas dominates. The latter possibility seems a priori more likely, given that the halo of ionized gas represents most of the volume, encloses most of the interstellar mass and is highly turbulent, but one cannot draw any definite conclusions without resorting to detailed numerical dynamo calculations. It is also noteworthy that, if the magnetic field in interstellar clouds is largely decoupled from that in the intercloud medium (as suggested by Morris \& Serabyn 1996), a local dynamo should naturally produce a dipolar field in the intercloud medium. Either way, a poloidal (and hence dipolar) large-scale magnetic field in the GC region could very well be explained by a local dynamo.

The different scenarios described above refer to the largescale component of the interstellar magnetic field in the intercloud medium near the GC. We emphasize that they are not mutually exclusive. In particular, it is quite possible (even likely, in our view) that inward advection from the Galactic disk, outflows from the Galactic nucleus and local dynamo amplification all came into play. We also note that the wide scatter observed in the orientations of NRFs, especially the smaller ones, is almost certainly due to interstellar turbulence, regardless of the exact mechanism responsible for the appearance of filaments.

We now turn to the magnetic field inside GC molecular clouds. According to Chuss et al. (2003), the reason why the field within dense clouds is approximately horizontal can be understood in two alternative ways. A first possibility would be that the GC region was originally pervaded by a large-scale azimuthal magnetic field, as could be the case if a strong toroidal field was generated in the accretion disk around the central black hole and then expelled horizontally into the surrounding ISM or if the field was amplified by a local dynamo dominated by the disk-like CMZ (see above). In this scenario, the original field direction would have been preserved only in the dense and massive molecular clouds; outside of them, it would have been distorted by winds due to supernovae. However, for the reasons outlined above, it is more likely that the GC region was originally pervaded by a large-scale poloidal magnetic field. In molecular clouds, this field would have been sheared out in a horizontal direction, either by the cloud bulk motions (from differential rotation or from turbulence) with respect to the diffuse intercloud medium (Novak et al. 2003; Chuss et al. 2003) or by the forces (of compressive or tidal nature) that created and/or shaped the clouds (Morris \& Serabyn 1996, and references therein).

Along different lines, Morris \& Serabyn (1996) suggested that the magnetic field within molecular clouds is only loosely coupled to the intercloud field (see also Morris 2007). Decoupling between cloud and intercloud fields is easily achieved if, as expected, clouds are rotating (e.g., Fletcher et al. 2009). Initially, clouds must be magnetically connected to the diffuse intercloud medium from which they form. However, as they contract and spin up, they rapidly wind up the field 
lines that thread them, until, following magnetic reconnection or ambipolar diffusion, the wound-up field inside them becomes detached from the external field. Since clouds generally rotate about roughly vertical axes, their final internal fields will be predominantly horizontal.

\subsection{Magnetic field strength: critical discussion}

At the present time, the most uncertain aspect of the large-scale interstellar magnetic field in the GC region is its strength, with current estimates ranging from $\sim 10 \mu \mathrm{G}$ to $\gtrsim 1 \mathrm{mG}$ in the diffuse ISM. The low values are observational estimates obtained for a supposed equipartition/minimum-energy state, while the high values are theoretical estimates based on pressure balance considerations. Both types of estimates involve some questionable assumptions and raise a number of problems, which we now briefly review and discuss, starting with the high- $B$ estimates.

The reasoning leading up to the picture of a pervasive $\mathrm{mG}$ magnetic field hinges on two separate arguments (see beginning of Sect. 3). First, the apparent resistance of NRFs to collisions with ambient molecular clouds implies $P_{\text {mag }} \gtrsim P_{\text {ram }}$, which translates into $B \gtrsim 1 \mathrm{mG}$, inside NRFs. Second, pressure confinement of NRFs requires $B \gtrsim 1 \mathrm{mG}$ in the external ISM as well. Let us examine both arguments in turn.

The first argument already presents some weaknesses. It is true that the majority of NRFs are nearly straight, even though a few of them appear to be significantly distorted and a few others could potentially have deformations that escape detection from Earth because of projection effects. A moot point, however, is whether NRFs are truly colliding with molecular clouds. Morris \& Serabyn (1996) pointed out that all the well-studied NRFs at the time of their writing showed definite signs (e.g., slight bending, brightness discontinuity) of physical interactions with adjacent molecular clouds. On the other hand, Yusef-Zadeh (2003) noted that dynamical collisions with molecular clouds should produce $\mathrm{OH}(1720 \mathrm{MHz})$ maser emission, yet none had been detected at the apparent interaction sites. In addition, some NRFs do not seem to have any molecular cloud associated with them (e.g., Lang et al. 1999a,b).

Another issue concerns the exact constraint imposed by the rigidity of a truly colliding NRF. Yusef-Zadeh \& Morris (1987b) derived the condition $P_{\text {mag }} \gtrsim P_{\text {ram }}$, and they adopted $n_{\mathrm{H}_{2}} \sim$ $10^{4} \mathrm{~cm}^{-3}$ to estimate $P_{\text {ram }}$. This value of $n_{\mathrm{H}_{2}}$ is probably too high, as the filling factor of molecular clouds with $n_{\mathrm{H}_{2}} \gtrsim 10^{4} \mathrm{~cm}^{-3}$ is only $\lesssim 3 \%$ (see Paper I) or even as low as $\sim 1 \%$ (Goto et al. 2008), so that the likelihood that a given NRF be colliding with such a high-density cloud is rather low. Most of the NRFs undergoing a cloud collision could actually be colliding with a lowerdensity cloud or with the low-density envelope of a dense cloud (Boldyrev, private communication). More importantly, the condition $P_{\text {mag }} \gtrsim P_{\text {ram }}$ itself may in fact be too stringent. Chandran (2001) showed that a filament interacting with a cloud at a single location along its length has only small distortions so long as the Alfvén speed in the intercloud medium, $V_{\mathrm{A}}=B / \sqrt{4 \pi \rho}$, is much greater than the cloud velocity, $v_{\text {cloud }}$. With $\rho=1.453 m_{\mathrm{p}} n_{\mathrm{H}}$, $\left\langle n_{\mathrm{H}}\right\rangle \sim(1-10) \mathrm{cm}^{-3}$ in the intercloud medium (see Paper I) and $v_{\text {cloud }} \sim 15 \mathrm{~km} \mathrm{~s}^{-1}$ (as in Yusef-Zadeh \& Morris 1987a,b), the easier condition $V_{\mathrm{A}} \gg v_{\text {cloud }}$ is equivalent to $B \gg(8-26) \mu \mathrm{G}$.

The bottom line is that for some NRFs, like those of the Radio Arc, which remain nearly straight despite multiple physical interactions with molecular clouds (Morris 1990), the original conclusion that $B \gtrsim 1 \mathrm{mG}$ seems reasonably solid. For other
NRFs, the evidence for a $\mathrm{mG}$ field is less compelling, or even completely absent.

The second argument is even more questionable. Morris (1990) reasoned that NRFs must be pressure-confined and, considering the possibility of plasma confinement, he estimated the thermal pressure of the very hot gas at $n T \sim 10^{7} \mathrm{~cm}^{-3} \mathrm{~K}$. Since this is much less than the pressure of a $1 \mathrm{mG}$ magnetic field, $P_{\text {mag }} / k \simeq 3 \times 10^{8} \mathrm{~cm}^{-3} \mathrm{~K}$, he concluded that the confining pressure cannot be thermal, but instead must be magnetic. This line of reasoning raises several important issues, some of which might possibly invalidate it.

First, Morris (1990) might have considerably underestimated the very hot gas thermal pressure. Koyama et al. (1996) presented X-ray spectroscopic imaging observations of a $1^{\circ} \times 1^{\circ}$ area around the GC, which point to a much higher value. Attributing the diffuse $\mathrm{X}$-ray continuum emission to thermal bremsstrahlung, they derived an electron temperature $k T_{\mathrm{e}} \gtrsim 10 \mathrm{keV}\left(T_{\mathrm{e}} \gtrsim 10^{8} \mathrm{~K}\right)$ and an electron density $n_{\mathrm{e}} \sim$ $(0.3-0.4) \mathrm{cm}^{-3}$. If ions and electrons are in collisional equilibrium, the corresponding total thermal pressure is $n T \gtrsim(6-8) \times$ $10^{7} \mathrm{~cm}^{-3} \mathrm{~K}$. But Koyama et al. (1996) found evidence that the thermal pressure of the ions might be at least one order of magnitude higher than that of the electrons. If this were to be confirmed, the very hot gas would be able to supply the required confining pressure for NRFs with $\mathrm{mG}$ fields. Interestingly, the $\simeq 270 \mathrm{pc}$ radial extent of the very hot gas region (Yamauchi et al. 1990) would then naturally explain why NRFs are observed over a $\sim 300$ pc region (LaRosa et al. 2004) only. However, we have to emphasize that the plasma pressure inferred from Koyama et al. (1996)'s work might be a little extreme. More recently, Muno et al. (2004) observed a smaller, $17^{\prime} \times 17^{\prime}$ area around the GC, with better signal-to-noise ratio and finer angular resolution. To explain the observed diffuse X-ray emission, they had to appeal to two plasma components: a soft component, with $k T \simeq 0.8 \mathrm{keV}$ and $n_{\mathrm{e}} \sim\left(0.1-0.5 \mathrm{~cm}^{-3}\right)(d / 50 \mathrm{pc})^{-1 / 2}$, and a hard component, with $k T \simeq 8 \mathrm{keV}$ and $n_{\mathrm{e}} \sim\left(0.1-0.2 \mathrm{~cm}^{-3}\right)(d / 50 \mathrm{pc})^{-1 / 2}$, where $d$ is the line-of-sight depth of the X-ray emitting region. Both components were assumed to be in collisional-ionization equilibrium, consistent with the measured energies and flux ratios of the spectral lines. Under these conditions and with $d \simeq 270 \mathrm{pc}$ (Yamauchi et al. 1990), the total thermal pressure of the plasma is $\sim(1-2) \times 10^{7} \mathrm{~cm}^{-3} \mathrm{~K}$, close to Morris (1990)'s original estimate and too low to confine NRFs. To be sure, the above pressure estimates make sense only to the extent that the observed diffuse $\mathrm{X}$-ray emission is truly produced by a thermal plasma. These estimates do not allow us to conclude either way on the potential ability of hot plasma to confine NRFs.

Another interesting possibility is that NRFs could be confined by magnetic tension forces (Lesch \& Reich 1992; Uchida \& Guesten 1995). Yusef-Zadeh \& Morris (1987b) noticed that the linear filaments of the Radio Arc appear to be surrounded by a helical magnetic structure winding about them. Having estimated that magnetic pressure in the filaments is much higher than the ambient gas pressure, they advanced the view that the magnetic field is in a local force-free state. Accordingly, the electric current flows along field lines, and the field-aligned current induces a locally toroidal field component, in agreement with the observed helical structure. This toroidal component, in turn, provides a confining magnetic tension force. Observational evidence for helical or twisted magnetic fields has been found in filaments other than those of the Radio Arc (e.g., Gray et al. 1995; Yusef-Zadeh et al. 1997; Morris et al. 2006). From a theoretical point of view, field-aligned currents and helical fields can be explained in various ways. Benford (1988) proposed an 
electrodynamic model for the Radio Arc, wherein the motion of a partially ionized molecular cloud across a strong poloidal magnetic field drives electric currents around a closed-loop circuit originating at the cloud and continuing along the poloidal field lines (see also Morris \& Yusef-Zadeh 1989). For the Snake, Bicknell \& Li (2001) envisioned the picture of a magnetic flux tube having both ends anchored in rotating molecular clouds; in this picture, the differential rotation between both clouds generates a locally toroidal field component and, hence, gives rise to a helical pattern. Each of these two models could potentially be applied to other NRFs, bearing in mind that twisted magnetic fields are subject to the kink instability. In any event, even if the operation of magnetic tension forces could be firmly established, it would still be difficult to quantify their actual contribution to the confinement of NRFs without a detailed knowledge of the exact magnetic configuration in and around NRFs.

Finally, and most importantly, NRFs do no need to be confined at all; they could very well be transient or dynamic structures out of mechanical balance with their surroundings. As a matter of fact, several plausible models for their origin describe them in these non-equilibrium terms. In the cometary-tail model of Shore \& Larosa (1999) ${ }^{9}$, NRFs are the long and thin magnetic wakes produced by a weakly magnetized Galactic wind impinging on GC molecular clouds. The advected magnetic field drapes around the clouds and stretches out behind them, growing to the point where its pressure balances the ram pressure of the Galactic wind. For typical wind parameters, the field inside the wakes thus reaches $\sim 1 \mathrm{mG}$, independent of the ambient field strength, which could be as low as $\sim 10 \mu \mathrm{G}$ (estimate based on the predicted wake lengths and on stability considerations). The overall orientation of the wakes is governed by the direction of the wind, which, the authors claim, is roughly vertical. Hence, the observed tendency of NRFs to run vertical would reflect the direction of the wind rather than that of the large-scale magnetic field (as usually presumed). An advantage of this dynamical model is that it avoids the MHD stability problems faced by static equilibrium models. It also provides an efficient mechanism to accelerate electrons to relativistic energies, through wave-particle interactions in the turbulent cascades driven near the current sheets of the wakes.

Still in the spirit of a dynamic ISM, NRFs could be regarded as direct manifestations of the intense turbulent activity characterizing the GC region (Boldyrev \& Yusef-Zadeh 2006). Turbulence there naturally leads to a highly intermittent magnetic field distribution, with strongly magnetized filamentary structures arising in an otherwise weak-field (e.g., $B \sim 10 \mu \mathrm{G}$ ) background. Because the turbulent intensity varies on a scale comparable to or only slightly larger than the outer scale of the turbulence, magnetic flux is expelled from the region of intense turbulence (diamagnetic pumping) before the field has a chance to grow strong everywhere. In this context, the observed NRFs would be nothing else than the strongly magnetized filaments, and their field strength, set by the external turbulent pressure, would typically be $\gtrsim 0.1 \mathrm{mG}$. The preferentially vertical direction of the longest NRFs could be related to the $\Omega$-shaped radio lobe detected by Sofue \& Handa (1984); more generally, it could be a direct consequence of the roughly poloidal geometry of the large-scale magnetic field. As for the synchrotron-emitting

\footnotetext{
9 Neither the cometary-tail model of Shore \& Larosa (1999) nor the turbulent model of Boldyrev \& Yusef-Zadeh (2006) is able to explain the Radio Arc, which differs from the other isolated NRFs in that it is clearly organized on a large scale.
}

electrons, they could be either pervasive or concentrated near localized sources.

Aside from the possible shortcomings in the derivation of a $\mathrm{mG}$ field, the claims for such a strong field have elicited a number of important criticisms. A frequently voiced objection is that the ensuing synchrotron lifetimes, $t_{\mathrm{syn}}$, are too short. The synchrotron lifetime is indeed a strongly decreasing function of field strength, given by $t_{\mathrm{syn}} \propto E^{-1} B_{\perp}^{-2}$ for a relativistic electron of energy $E$ and by $t_{\mathrm{syn}} \propto v^{-1 / 2} B_{\perp}^{-3 / 2}$ at the corresponding synchrotron frequency, $v \propto B_{\perp} E^{2}$. At the $74 \mathrm{MHz}$ and $330 \mathrm{MHz}$ frequencies of the diffuse nonthermal emission detected by LaRosa et al. (2005), the synchrotron lifetimes in a $B_{\perp}=1 \mathrm{mG}$ field would only be $t_{\mathrm{syn}} \simeq 1.2 \times 10^{5} \mathrm{yr}$ and $t_{\mathrm{syn}} \simeq 0.6 \times 10^{5} \mathrm{yr}$, respectively. Unless the source of nonthermal emission is shortlived, relativistic electrons would need to be injected or reaccelerated on such short timescales. LaRosa et al. (2005) considered this to be implausible, whereas Morris (2007) estimated that the local supernova rate is more than sufficient to meet the required timescales.

At the higher $1.5 \mathrm{GHz}$ and $5 \mathrm{GHz}$ frequencies of many NRF observations, the synchrotron lifetimes in a $1 \mathrm{mG}$ field would be even shorter: $t_{\mathrm{syn}} \simeq 2.7 \times 10^{4} \mathrm{yr}$ and $t_{\mathrm{syn}} \simeq 1.5 \times$ $10^{4} \mathrm{yr}$, respectively (supposing $B_{\perp} \simeq B$, a reasonable assumption in the case of NRFs). Here, the trouble is not so much that these short lifetimes would impose similarly short injection/reacceleration timescales - as explained above, NRFs could be short-lived structures, and if they are not, one could easily imagine that they are fueled by local, long-lived sources of relativistic electrons. The real concern is that electrons might not be able to travel far enough (e.g., Yusef-Zadeh 2003; Morris 2007). Presumably, relativistic electrons stream along field lines at about the Alfvén speed in the ionized gas, $V_{\mathrm{A}, \mathrm{i}}=B / \sqrt{4 \pi \rho_{i}}$, such that, over their lifetimes $t_{\mathrm{syn}}$, they travel distances $d \simeq$ $V_{\mathrm{A}, \mathrm{i}} t_{\mathrm{syn}} \propto v^{-1 / 2} \rho_{i}^{-1 / 2} B^{-1 / 2}$. The ionized gas density, $\rho_{i}$, is quite uncertain, as witnessed by the widely different values adopted by different authors. Since the ionized phase of the ISM is largely dominated by its warm component, where helium is only weakly ionized, we may let $\rho_{i} \simeq m_{\mathrm{p}} n_{\mathrm{e}}$. If we further approximate the free-electron density, $n_{\mathrm{e}}$, by its space-averaged value from Cordes \& Lazio (2002)'s model, we find that $n_{\mathrm{e}} \simeq 10 \mathrm{~cm}^{-3}$ close to the GC and $n_{\mathrm{e}} \gtrsim 1 \mathrm{~cm}^{-3}$ out to $\simeq 220 \mathrm{pc}$ along the Galactic plane and up to $\simeq 40 \mathrm{pc}$ along the vertical, i.e., in most of the region of interest. For a $1 \mathrm{mG}$ field, the Alfvén speed is $V_{\mathrm{A}, \mathrm{i}} \simeq\left(2200 \mathrm{~km} \mathrm{~s}^{-1}\right)\left(n_{\mathrm{e}} / 1 \mathrm{~cm}^{-3}\right)^{-1 / 2}$ and the traveled distances at $1.5 \mathrm{GHz}$ and $5 \mathrm{GHz}$ are $d \simeq(60 \mathrm{pc})\left(n_{\mathrm{e}} / 1 \mathrm{~cm}^{-3}\right)^{-1 / 2}$ and $d \simeq(33 \mathrm{pc})\left(n_{\mathrm{e}} / 1 \mathrm{~cm}^{-3}\right)^{-1 / 2}$, respectively. These calculated distances are compatible with the observed lengths of most NRFs, but they are difficult to reconcile with some NRFs being as long as $\sim 60 \mathrm{pc}$. This difficulty can be circumvented if the longest NRFs lie in a particularly low- $n_{\mathrm{e}}$ environment, or if particle injection occurs at more than one location along them (for instance, the Snake, which is $\sim 60$ pc long, has two kinks along its length, one or both of which could conceivably be associated with particle injection; e.g., Bicknell \& Li 2001), or else if reacceleration takes place more-or-less continuously along them (e.g., Morris 2007). Incidentally, re-acceleration also provides a possible explanation for the observed constancy of the radio spectral index along the lengths of several NRFs (e.g., Lang et al. 1999b; LaRosa et al. 2000), although it is not immediately clear why re-acceleration would precisely counteract the spectral steepening due to synchrotron losses.

The above discrepancy between the calculated distances traveled by relativistic electrons in a $1 \mathrm{mG}$ field and the observed 
lengths of the longest NRFs is only marginal. Furthermore, there exist viable ways, other than decreasing the field strength, to bring them into agreement. But more fundamentally, the discrepancy poses a problem only to the extent that NRFs are "illuminated flux tubes", i.e., flux tubes into which relativistic electrons have been injected. Should they instead be regions of compressed magnetic field (e.g., Shore \& Larosa 1999; Boldyrev \& Yusef-Zadeh 2006), the mismatch would become totally irrelevant. For all these reasons, we feel that the short synchrotron lifetimes and the associated short distances traveled by relativistic electrons may not be held up against a $\mathrm{mG}$ field inside NRFs.

To conclude our discussion of the high- $B$ estimates, the claim that NRFs have a $\mathrm{mG}$ field may have to be toned down. While there is good evidence that a fraction of them do, others could possibly have a weaker field. As for the general ISM, we see no cogent reason to believe that it is magnetized at the $\mathrm{mG}$ level.

We now turn to the low- $B$ estimates and discuss the validity of the equipartition/minimum-energy assumption, which, for brevity, we will refer to as the equipartition assumption. This hypothesis admits no rigorous theoretical justification, yet it seems to yield acceptable results in large-scale regions of the Galactic disk as well as in external galaxies as wholes (e.g., Beck 2001; Beck \& Krause 2005). In these objects, the large-scale magnetic field is predominantly horizontal, the total field strength is typically a few $\mu \mathrm{G}$ and cosmic-ray sources (e.g., supernova shocks) likely abound. As a result, vast amounts of cosmic rays are injected into the ISM, where the horizontal field lines tend to keep them confined. Cosmic-ray pressure can then build up until it reaches a value, presumably $\sim P_{\text {mag }}$, that is high enough to break the magnetic confinement - for instance, via the Parker instability. Not only does this instability enable cosmic rays to escape from the galactic disk through the formation and rupture of giant magnetic loops, but it also leads to magnetic field amplification through enhanced dynamo action (Parker 1992; Hanasz et al. 2004, 2009). Both effects conspire to maintain cosmic-ray pressure comparable to magnetic pressure.

The situation near the GC is completely different. Cosmicray sources are undoubtedly much more abundant there than in the Galactic disk. On the other hand, the large-scale magnetic field is approximately vertical, so that cosmic rays streaming along field lines naturally follow the shortest path out of the Galaxy (Chandran et al. 2000). In addition, if the field is as strong as $\sim 1 \mathrm{mG}$, cosmic rays stream away at a very high speed $\left(\sim V_{\mathrm{A}, \mathrm{i}}\right) .{ }^{10}$ Thus, both the injection and the escape of cosmic rays proceed at a much faster rate than in the disk. More to the point, the two processes are not related by a self-regulating mechanism, such as the "cosmic-ray valve" operating in the disk, which would, at the same time, keep cosmic-ray and magnetic pressures close to equipartition.

Observationally, the obvious rigidity and organized structure of NRFs strongly support the idea that they are magnetically dominated (e.g., Anantharamaiah et al. 1991; Lang et al. 1999b). The case of the general ISM is not so self-evident. Observations of the diffuse $\gamma$-ray emission from the GC region reveal a pronounced $\gamma$-ray excess within $\sim 0.6$ of the GC (Mayer-Hasselwander et al. 1998), which could speak in favor of an elevated cosmic-ray pressure there. However, it is unlikely

\footnotetext{
10 Regardless of the field strength, it is likely that the powerful winds emanating from the GC (e.g., Bland-Hawthorn \& Cohen 2003) or produced by supernova explosions also contribute to cosmic-ray escape. However, since these winds tend to evacuate magnetic fields as well, it is not clear that their net effect is to reduce cosmic-ray pressure relative to magnetic pressure.
}

that this $\gamma$-ray excess can entirely be attributed to cosmic-ray interactions with interstellar matter. In fact, Mayer-Hasselwander et al. (1998) showed that their $\gamma$-ray data could be reproduced with a combination of unresolved compact sources (such as pulsars) and a truly diffuse interstellar contribution from cosmic rays having the same density as in the inner Galaxy. This result, combined with LaRosa et al. (2005)'s study of the diffuse nonthermal radio emission from the GC region, lends some credence to the equipartition assumption.

To conclude our discussion of the low- $B$ estimates, NRFs probably have super-equipartition magnetic fields, whereas the general ISM might have its magnetic field in rough equipartition with cosmic rays.

New observations with the Fermi gamma-ray space telescope will hopefully shed more light on this topic, although using the Fermi data to obtain better constraints on the cosmic-ray electron energy density near the GC might prove rather tricky. In principle, the task will require: (1) removing the foreground $\gamma$ ray emission along the long line of sight toward the GC region; (2) isolating the diffuse interstellar emission produced by cosmic rays; (3) separating the contributions from cosmic-ray nuclei ( $\pi^{0}$ decay) and electrons (bremsstrahlung and inverse Compton scattering); and (4) having a good knowledge of both the interstellar matter distribution (for the $\pi^{0}$ decay and bremsstrahlung components) and the interstellar radiation field (for the inverse Compton component). One difficulty for cosmic-ray electrons is that their contribution to $\gamma$-ray emission in the energy range covered by Fermi (ideally $\sim 30 \mathrm{MeV}-300 \mathrm{GeV}$ ) is overshadowed by the contribution from cosmic-ray nuclei. On the other hand, the inverse Compton component, observed slighty above or below the Galactic plane, is expected to be less contaminated by foreground emission, insofar as the photon density is much higher in the Galactic bulge than along the line of sight (Jean Ballet, private communication). Altogether, there is good hope that Fermi observations of the inverse Compton emission from the GC region will help to determine the cosmic-ray electron density with better accuracy, and, when coupled with the low-frequency radio continuum data, help to constrain the magnetic field strength near the GC.

Faraday rotation studies do not add any significant constraints on the interstellar magnetic field strength. The inferred values of $\left|B_{\|}\right|$are typically a few $\mu \mathrm{G}$, with considerable uncertainty (see Sect. 3.2). These values of $\left|B_{\|}\right|$indicate that the largescale magnetic field in the diffuse ionized medium is $\gtrsim$ a few $\mu \mathrm{G}$, which is compatible with both an equipartition field $\sim 10 \mu \mathrm{G}$ and a dynamically dominant field $\sim 1 \mathrm{mG}$. However, if the large-scale field has a roughly poloidal geometry, with only a small component along the line of sight, the equipartition estimate might be a little too low to explain the Faraday rotation results.

For dense neutral clouds, Zeeman splitting studies lead to a mixture of positive detections, with $\left|B_{\|}\right| \sim(0.1-1) \mathrm{mG}$ (outside the Sgr A region), and non-detections, with $\left|B_{\|}\right| \lesssim(0.1-1) \mathrm{mG}$, again subject to important uncertainty as well as possible dilution of the Zeeman signal (see Sect. 3.4). These mixed results can be understood if the magnetic field inside dense clouds is roughly horizontal, such that its line-of-sight component may lie anywhere between 0 and the total field strength. The latter could then be $\sim 1 \mathrm{mG}$ or loosely range from a few $0.1 \mathrm{mG}$ to a few $\mathrm{mG}$. A less direct, and even cruder, estimation of the field strength inside molecular clouds was made by Chuss et al. (2003), based on their Smm polarimetric observations. Interpreting the measured dependence of field direction on gas density in terms of shearing of an initially poloidal field, they came up with a characteristic field strength $\sim 3 \mathrm{mG}$ (see Sect. 3.3). 


\section{Additional input}

\subsection{Connection with the rest of the Galaxy}

How does our view of the interstellar magnetic field in the GC region fit in with what we know about the magnetic field in the Galaxy at large? Are both magnetic systems connected in any way or are they completely independent?

Our current knowledge of the overall distribution and morphology of the Galactic magnetic field away from the GC relies primarily on synchrotron emission and Faraday rotation studies.

Synchrotron intensity measurements give access to the total field strength distribution, subject to the assumption of equipartition between magnetic fields and cosmic rays (discussed in Sect. 4.2). Based on the synchrotron map of Beuermann et al. (1985), Ferriere (1998) thus found that the total field has a value $\simeq 5 \mu \mathrm{G}$ near the Sun, a radial scale length $\simeq 12 \mathrm{kpc}$ and a local vertical scale height $\simeq 4.5 \mathrm{kpc}$. In addition, synchrotron polarimetry indicates that the local ratio of ordered (regular + anisotropic random) to total fields is $\simeq 0.6$ (Beck 2001), implying an ordered field $\simeq 3 \mu \mathrm{G}$ near the Sun.

Faraday rotation measures of Galactic pulsars and extragalactic radio sources also provide valuable information, more specifically relevant to the uniform (or regular) magnetic field, $\mathbf{B}_{\mathrm{u}}$, in the ionized ISM. Here is a summary of what we have learnt from them, first on the strength and second on the direction of $\mathbf{B}_{\mathrm{u}}$. From pulsar RMs, we now know that $B_{\mathrm{u}} \simeq 1.5 \mu \mathrm{G}$ near the Sun (Rand \& Kulkarni 1989; Han et al. 2006) and that $B_{\mathrm{u}}$ increases toward the GC, to $\gtrsim 3 \mu \mathrm{G}$ at $r=3 \mathrm{kpc}$ (Han et al. $2006)$, i.e., with an exponential scale length $\$ 7.2 \mathrm{kpc}$. $B_{\mathrm{u}}$ also decreases away from the Galactic plane, albeit at a very uncertain rate - extragalactic-source RMs suggest an exponential scale height $\sim 1.4 \mathrm{kpc}$ (Inoue \& Tabara 1981).

In the Galactic disk, $\mathbf{B}_{\mathrm{u}}$ is nearly horizontal and generally dominated by its azimuthal component. Near the Sun, $\mathbf{B}_{\mathrm{u}}$ points toward $l \simeq 82^{\circ}$, corresponding to a magnetic pitch angle $p \simeq-8^{\circ}$ (Han et al. 1999) and implying a clockwise direction about the $z$-axis (see Fig. 1). However, $\mathbf{B}_{\mathrm{u}}$ reverses several times with decreasing radius, the number and radial locations of the reversals being still highly controversial (Rand \& Lyne 1994; Han et al. 1999; Vallée 2005; Han et al. 2006; Brown et al. 2007; Noutsos et al. 2008). These reversals have often been interpreted as evidence that the uniform field is bisymmetric (azimuthal wavenumber $m=1)$, although an axisymmetric $(m=0)$ field would be expected from dynamo theory. Recently, Men et al. (2008) showed that neither the axisymmetric nor the bisymmetric picture is consistent with the existing pulsar RMs, and they concluded that the uniform field must have a more complex pattern. Along the vertical, $\mathbf{B}_{\mathrm{u}}$ is roughly symmetric in $z,{ }^{11}$ at least close enough to the midplane (Rand \& Lyne 1994).

In the Galactic halo, $\mathbf{B}_{\mathrm{u}}$ could have a significant vertical component, with $\left(B_{\mathrm{u}}\right)_{z} \sim 0.37 \mu \mathrm{G}$ (Han et al. 1999) or $\left(B_{\mathrm{u}}\right)_{z} \sim$ $0.25 \mu \mathrm{G}$ on average between the North and South Poles (Sui et al. 2009) at the position of the Sun. In contrast to the situation in the disk, the azimuthal component of $\mathbf{B}_{\mathrm{u}}$ shows no sign of reversal with decreasing radius. Along the vertical, $\mathbf{B}_{\mathbf{u}}$ is roughly antisymmetric in $z$ (counterclockwise at $z>0$ and clockwise at $z<0$ ) inside the solar circle and roughly symmetric (clockwise at all $z$ ) outside the solar circle (Han et al. 1997, 1999).

\footnotetext{
11 A magnetic field is said to be symmetric (antisymmetric) in $z$, or, equivalently, quadrupolar (dipolar), if its horizontal component is an even (odd) function of $z$ and its vertical component an odd (even) function of $z$.
}

Sun et al. (2008) developed comprehensive 3D models of the Galactic magnetic field, constrained by an all-sky map of extragalactic-source RMs together with observations of the Galactic total and polarized emission over a wide range of radio frequencies. They obtained a good fit to the data for axisymmetric models where the disk field is purely horizontal, has constant pitch angle $p=-12^{\circ}$, reverses inside the solar circle and is symmetric in $z$ (clockwise near the Sun), while the halo field is purely azimuthal and antisymmetric in $z$ (counterclockwise/clockwise above/below the midplane at all radii). They also came to the conclusion that bisymmetric models are incompatible with the RM data.

Finding $\mathbf{B}_{\mathrm{u}}$ to have quadrupolar parity in the disk and dipolar parity in the halo is consistent with the predictions of dynamo theory and with the results of galactic dynamo calculations, even if, in the very long run (i.e., on timescales longer than current galactic ages), the field may eventually evolve toward a singleparity state (Brandenburg et al. 1992; Ferrière \& Schmitt 2000; Moss \& Sokoloff 2008). Moreover, the signs of $\left(B_{\mathrm{u}}\right)_{r} /\left(B_{\mathrm{u}}\right)_{\theta}$ in the disk (negative throughout) and $\left(B_{\mathrm{u}}\right)_{z} /\left(B_{\mathrm{u}}\right)_{\theta}$ in the halo (negative/positive above/below the midplane) are those expected from azimuthal shearing of radial and vertical fields, respectively, by the large-scale differential rotation of the Galaxy.

Let us now look into the possible connections between the Galactic magnetic field described above and the GC field discussed in the previous sections. Here, we are only interested in the uniform component of the field, which, for brevity, we will simply refer to as the field. As explained at the beginning of Sect. 3, the observed orientations of NRFs suggest that the GC field is approximately poloidal, and hence dipolar, in the diffuse intercloud medium. However, they do not tell us whether the field is pointing north or south.

Faraday rotation studies could, under certain conditions, provide the sought-after sign information, but unfortunately they lead to conflicting conclusions. Novak et al. (2003) noted that the available RMs toward NRFs within $1^{\circ}$ of the GC exhibit a sign pattern indicative of an antisymmetric field running counterclockwise above the midplane and clockwise below it (see Sect. 3.2) - exactly as in the Galactic halo. They further suggested that this pattern could result from azimuthal shearing by the Galactic differential rotation of an initially vertical field pointing north $\left(B_{z}>0\right)$ - again as in the halo. In contrast, Roy et al. (2008) found that the RMs of background extragalactic sources seen through the area $\left(|l|<6^{\circ},|b|<2^{\circ}\right)$ are mostly positive in each of the four quadrants, consistent with a symmetric field pointing toward us. Such a symmetric field cannot be produced by large-scale shearing, or by any symmetric distortion, of a poloidal field. It is, therefore, probably unrelated to the dominant poloidal field and may not be used to constrain its (north or south) direction.

Zeeman splitting studies are not of great help here. The only true detections outside the $\mathrm{Sgr}$ A region and away from $\mathrm{OH}$ masers pertain to Sgr B2, where Crutcher et al. (1996) measured $B_{\|}<0$ (see Sect. 3.4 and footnote 7). If the line-of-sight field in the dense clouds sampled by Zeeman measurements has the same sign as in the surrounding intercloud medium, Crutcher et al. (1996)'s results suggest RM > 0 toward Sgr B2. Since Sgr B2 lies in the $(l>0, b>0)$ quadrant, this RM sign is in agreement with the general RM patterns of both Novak et al. (2003) and Roy et al. (2008).

Irrespective of the exact RM pattern and of the sign of $B_{z}$ near the GC, the predominantly poloidal, and hence dipolar, GC field could naturally connect with the dipolar halo field. Both fields together could actually form a single magnetic system, 
which, in turn, could be the outcome of a large-scale quasispherical dynamo - corresponding, for instance, to an A0 (antisymmetric \& $m=0$ ) dynamo mode (Han et al. 1997). However, one may not jump to the conclusion that the poloidal field is a pure dipole, as proposed by Han (2002) and often assumed in the cosmic-ray propagation community (e.g., Alvarez-Muñiz et al. 2002; Prouza \& Šmída 2003). A pure dipole can be expected in a current-free medium, but not inside the highly conducting ISM. Even the superposition of a pure dipole and an azimuthal field is unlikely here, for several reasons. From a theoretical point of view, this particular combination would rule out any ring current. Moreover, numerical simulations of galactic dynamos always yield more complex magnetic geometries. From an observational point of view, if the poloidal field near the GC were a pure dipole, NRFs crossing the midplane would be curved inward, whereas, on the whole, they exhibit a slight outward curvature (Morris 1990). Besides, the NRF spatial distribution displayed in Fig. 29 of Yusef-Zadeh et al. (2004) does not at all convey the sense of a pure dipole. Finally, as noted by Han (2002) himself, a global dipole with $B_{z}>0$ at the position of the Sun should have $B_{z}<0$ near the GC, which is exactly opposite to Novak et al. (2003)'s finding. The matter is in fact a little more subtle, as a dipole field may have either sign of $B_{z}$ near the GC, depending on Galactic polar angle, $\Theta$ (for reference, $B_{z} \gtrless 0$ for $\Theta \gtrless \Theta_{\text {crit }}$, where $\left.\Theta_{\text {crit }}=\arccos (1 / \sqrt{3})=54^{\circ} .7\right)$. Anyway, a more realistic guess would be that the poloidal field, out to beyond the solar circle, is everywhere pointing north and slightly curved outward, which would be consistent with the inward advection scenario.

The azimuthal component of the GC field, revealed through Faraday rotation, does not have a well-established parity. If it has dipolar parity (as suggested by Novak et al. 2003), it is probably directly coupled to the dominant poloidal component and, together with it, connected to the dipolar halo field. On the other hand, if the azimuthal component has quadrupolar parity (as suggested by Roy et al. 2008), it is probably decoupled from the poloidal component, but it could connect with the quadrupolar disk field if the latter is bisymmetric, or at least possesses a bisymmetric mode. If the quadrupolar disk field is axisymmetric or contains an axisymmetric mode, the associated poloidal field lines cannot remain horizontal all the way in to the GC, but they have to diverge vertically somewhere before reaching the GC region. They can then, on each side of the Galactic plane, turn around in the halo and arc back to the disk, thereby forming one large or several smaller magnetic loops (e.g., Ferrière \& Schmitt 2000).

To close up the discussion, let us mention the possibility that the GC region could harbor its own magnetic system, independent of the magnetic field pervading the Galaxy at large. The origin of such a separate magnetic system could be a GC dynamo, possibly modified by outflows from the nucleus.

\subsection{Clues from external galaxies}

Could observations of external galaxies shed some light on the properties of the interstellar magnetic field near the GC? External galaxies do not give access to the wealth of details that can be detected in our own Galaxy, but they provide a global view which can help fill in some of the gaps in the picture of the Galactic magnetic field.

The observational status of interstellar magnetic fields in external spiral galaxies was recently reviewed by Beck (2008) and Krause (2009). For practical purposes, external galaxies are generally considered as either face-on (if they are not or mildly inclined) or edge-on (if they are strongly inclined). While both groups bring along complementary pieces of information, the tools used to study them are identical and the same as for our Galaxy, namely, synchrotron emission and Faraday rotation.

Face-on galaxies are ideally suited to determine the strength and the horizontal structure of magnetic fields in galactic disks. Total field strengths, derived from measurements of the synchrotron total intensity together with the equipartition assumption, are $\sim 10 \mu \mathrm{G}$ on average - more precisely, $\sim 5 \mu \mathrm{G}$ in radio-faint galaxies, $\sim 15 \mu \mathrm{G}$ in more active (higher starformation rate) galaxies and up to $\sim(50-100) \mu \mathrm{G}$ in starburst galaxies. Ordered field strengths, derived from measurements of the synchrotron polarized intensity, are $\sim(1-5) \mu \mathrm{G}$ on average. The total field is always strongest in the optical spiral arms, where it can reach up to $\sim(20-30) \mu \mathrm{G}$ (for normal galaxies), whereas the ordered field is generally somewhat stronger in the interarm regions, where it can reach up to $\sim(10-15) \mu \mathrm{G}$. Finally, according to synchrotron polarization maps, the ordered field tends to follow the orientation of the optical spiral arms, such that magnetic pitch angles are typically $\sim 10^{\circ}-40^{\circ}$ (in absolute value).

Edge-on galaxies are best suited to determine the vertical structure of magnetic fields in galactic disks and halos. Most of them appear to possess extended synchrotron halos, the vertical size of which implies that the total field has a vertical scale height $\sim 7 \mathrm{kpc}$, with little scatter amongst galaxies. Since the polarization degree increases away from the midplane, the ordered field probably has an even greater vertical scale height. Polarization maps indicate that the ordered field is generally nearly horizontal close to the midplane. For galaxies with highsensitivity measurements (e.g., NGC 891, NGC 5775, NGC 253, M 104), the ordered field becomes more vertical in the halo, with $\left|B_{z}\right|$ increasing with both $|z|$ and $r$. The resulting X-shaped field is extremely different from the dipole-like field that might be expected from dynamo theory (see Sect. 5.1), not only along the rotation axis, where it goes horizontal instead of vertical, but also at large distances from the center, where it diverges from the midplane instead of curving back to it. One possible theoretical explanation for the existence of such X-shaped halo fields involves galactic winds with roughly radial streamlines (see, e.g., Dalla Vecchia \& Schaye 2008).

The one notable exception amongst edge-on galaxies is NGC 4631. In the disk of this galaxy, the ordered field runs nearly vertical throughout the innermost $\sim 5 \mathrm{kpc}$, and it is only at larger radii that it turns roughly horizontal. In the halo, the ordered field has a more radial appearance, which, away from the rotation axis, bears some resemblance to the $\mathrm{X}$ shape observed in other edge-on galaxies. The unusual magnetic pattern of NGC 4631 could perhaps be related to the existence of a central starburst and/or to the large-scale rotation being almost rigid inside $\sim 5 \mathrm{kpc}$.

As in our Galaxy, the measured synchrotron polarization angles only give the orientation of the ordered magnetic field, not its direction. The field direction can sometimes be gathered from Faraday rotation measures (of either the galactic synchrotron emission itself or background radio sources). This is how, by combining the observed polarization angles and rotation measures, it has been possible to identify well-defined azimuthal dynamo modes in a (small) number of galaxies. For instance, a dominating axisymmetric spiral mode was inferred in the disks of M 31 and IC 342, while a dominating bisymmetric spiral mode was suggested for the disk of M 81. Somewhat intriguingly, though, very few field reversals have been detected in 
the disks of external galaxies (Beck 2001), in contrast to what might be expected from the situation in our Galaxy. Regarding the vertical parity, evidence was found for a symmetric field in the disks of NGC 891 and IC 342.

Observations of external galaxies have a typical spatial resolution of a few 100 pc to a few kpc (Marita Krause, private communication). Evidently, this resolution is way too low to enable detection of strongly magnetized filaments similar to the NRFs observed near the GC. Even a large-scale vertical field confined to the innermost $\sim 300 \mathrm{pc}$ would probably escape detection. In other words, external galaxies could possibly host vertical fields near their centers, but (with the exception of NGC 4631) such vertical fields could not extend beyond the central kpc or so. Moreover, in none of the edge-on galaxies observed so far (neither in those featuring an X-shaped field nor in NGC 4631) does the halo field resemble a dipole.

Ultimately, what can be retained from observations of external galaxies is the following: If our Galaxy does not differ too much from the nearby galaxies whose magnetic fields have been mapped out, one may conclude that the vertical field detected close to the GC is only local, i.e., restricted to a region smaller than $\sim 1 \mathrm{kpc}$. One can then imagine two different possibilities: either the GC field is completely separate from the general Galactic field, or else it merges smoothly with the poloidal halo field, though not in the form of an approximate dipole.

\section{Conclusions}

Based on all the observational evidence presented in Sect. 3, we may be reasonably confident that the interstellar magnetic field in the GC region is approximately poloidal on average in the diffuse intercloud medium and approximately horizontal in dense interstellar clouds. Direct measurements of field strengths are scanty and altogether not very informative. However, our critical discussion of the existing observational and theoretical estimations in Sect. 4.2 prompts us to conclude, at least tentatively, that the general ISM is pervaded by a relatively weak magnetic field $(B \sim 10 \mu \mathrm{G})$, close to equipartition with cosmic rays $\left(B_{\mathrm{eq}} \sim 10 \mu \mathrm{G}\right)$, and that it contains a number of localized filamentary structures (the observed NRFs) with much stronger fields (up to $B \sim 1 \mathrm{mG}$ ), clearly above equipartition $\left(B_{\text {eq }} \sim 100 \mu \mathrm{G}\right)$. In dense interstellar clouds, the field would be a few $0.1 \mathrm{mG}$ to a few $\mathrm{mG}$ strong.

In our view, the high- $B$ filamentary structures are probably dynamic in nature. They could, for instance, have a turbulent origin, as suggested by Boldyrev \& Yusef-Zadeh (2006). If the GC region is the open magnetic system envisioned by these authors, turbulent dynamo action there should produce strongly magnetized filaments, with $P_{\text {mag }} \sim P_{\text {turb }}$, i.e., according to our estimation in Sect. 4.1, $B \sim 0.2 \mathrm{mG}$. Of course, there would be a whole range of field strength, allowing some filaments to have $B \gtrsim 1 \mathrm{mG}$. Besides, other mechanisms could also come into play, such as the formation of magnetic wakes behind molecular clouds embedded in a Galactic wind (Shore \& Larosa 1999). The higher ram pressure associated with the wind could then account for a larger number of filaments with $\mathrm{mG}$ fields.

It is interesting to note that if all NRFs have $B \sim 1 \mathrm{mG}$, the synchrotron data can be reproduced with a roughy uniform (within a factor $\sim 10$ ) relativistic-electron density, $n_{\mathrm{e}}$. Indeed, the factor $\sim 10$ between the equipartition field strengths inside NRFs $\left(B_{\text {eq }} \sim 100 \mu \mathrm{G}\right)$ and in the general ISM $\left(B_{\text {eq }} \sim 10 \mu \mathrm{G}\right)$ translates into a factor $\sim 100$ between their respective equipartition electron densities. Now, since synchrotron emissivity is $\propto n_{\mathrm{e}} B_{\perp}^{1-\alpha} v^{\alpha}$, the synchrotron emission of NRFs can equally be explained by the equipartition values $B \sim B_{\text {eq }} \sim 100 \mu \mathrm{G}$ and $n_{\mathrm{e}} \sim n_{\mathrm{e}, \mathrm{eq}}$ or by the magnetically dominated values $B \sim 1 \mathrm{mG} \sim 10 B_{\text {eq }}$ and $n_{\mathrm{e}} \sim 10^{\alpha-1} n_{\mathrm{e}, \mathrm{eq}}$. The synchrotron spectral index, $\alpha$, varies considerably amongst NRFs, from $\sim-2$ to $\sim+0.4$, with $\alpha \sim-0.6$ being quite typical (e.g., Reich et al. 1988; Anantharamaiah et al. 1991; Gray et al. 1995; Lang et al. 1999a,b; LaRosa et al. 2000, 2004). For NRFs with decreasing spectra $(\alpha<0)$, a mG field then implies an electron density $\sim 10-1000$ times lower than the equipartition value, i.e., within a factor $\sim 10$ of the equipartition electron density in the general ISM.

The situation with highly variable magnetic field and roughly uniform relativistic-electron density is in sheer contrast with the conventional picture of a uniformly strong magnetic field. Qualitatively, the former could be understood as the net result of two antagonistic effects: On the one hand, when a high- $B$ filament forms by compression, the attached electrons are compressed together with the field lines. On the other hand, once the electrons find themselves in a high- $B$ filament, they cool off (via synchrotron radiation) more rapidly and they stream away along field lines (at about the Alfvén speed) faster than electrons in the surrounding medium.

In reality, both the picture of a uniformly strong magnetic field and the picture of a roughly uniform relativistic-electron density are probably too extreme. It is much more likely that the $\mathrm{GC}$ region resides in an intermediate state, where both the field strength and the electron density are higher in NRFs than in the general ISM. Such a state is automatically achieved if all NRFs have a field strength comprised between the equipartition value $\left(B_{\text {eq }} \sim 100 \mu \mathrm{G}\right)$ and $B \sim 1 \mathrm{mG}$.

Acknowledgements. The author would like to thank J. Ballet, R. Beck, S. Boldyrev, R. Crutcher, M. Hanasz, J. Knödlseder, M. Krause, A. Marcowith, F. Martins, I. Moskalenko, G. Novak, W. Reich, A. Shukurov, A. Strong, X. Sun and the referee, T. LaRosa, for their valuable comments and detailed answers to her questions.

\section{References}

Aitken, D. K., Briggs, G. P., Roche, P. F., Bailey, J. A., \& Hough, J. H. 1986, MNRAS, 218, 363

Aitken, D. K., Smith, C. H., Gezari, D., McCaughrean, M., \& Roche, P. F. 1991, ApJ, 380, 419

Alvarez-Muñiz, J., Engel, R., \& Stanev, T. 2002, ApJ, 572, 185

Anantharamaiah, K. R., Pedlar, A., Ekers, R. D., et al. 1991, MNRAS, 249, 262

Beck, R. 2001, Space Sci. Rev., 99, 243

Beck, R. 2008, in AIP Conf. Ser., 1085, 83

Beck, R., \& Krause, M. 2005, Astron. Nachr., 326, 414

Benford, G. 1988, ApJ, 333, 735

Beuermann, K., Kanbach, G., \& Berkhuijsen, E. M. 1985, A\&A, 153, 17

Bicknell, G. V., \& Li, J. 2001, ApJ, 548, L69

Bland-Hawthorn, J., \& Cohen, M. 2003, ApJ, 582, 246

Boldyrev, S., \& Yusef-Zadeh, F. 2006, ApJ, 637, L101

Bower, G. C., Backer, D. C., \& Sramek, R. A. 2001, ApJ, 558, 127

Brandenburg, A., Donner, K. J., Moss, D., et al. 1992, A\&A, 259, 453

Brown, J. C., Haverkorn, M., Gaensler, B. M., et al. 2007, ApJ, 663, 258

Chakrabarti, S. K., Rosner, R., \& Vainshtein, S. I. 1994, Nature, 368, 434

Chandran, B. D. G. 2001, ApJ, 562, 737

Chandran, B. D. G., Cowley, S. C., \& Morris, M. 2000, ApJ, 528, 723

Chevalier, R. A. 1992, ApJ, 397, L39

Chuss, D. T., Davidson, J. A., Dotson, J. L., et al. 2003, ApJ, 599, 1116

Cordes, J. M., \& Lazio, T. J. W. 2002, ArXiv Astrophysics e-prints

Crutcher, R. M., Roberts, D. A., Mehringer, D. M., et al. 1996, ApJ, 462, L79

Dalla Vecchia, C., \& Schaye, J. 2008, MNRAS, 387, 1431

Davidson, J. A. 1996, in Polarimetry of the Interstellar Medium, ed. W. G. Roberge, \& D. C. B. Whittet, ASP Conf. Ser., 97, 504

Eckart, A., Genzel, R., Hofmann, R., Sams, B. J., \& Tacconi-Garman, L. E. 1995, ApJ, 445, L23

Ferriere, K. 1998, ApJ, 497, 759

Ferrière, K., Gillard, W., \& Jean, P. 2007, A\&A, 467, 611

Ferrière, K., \& Schmitt, D. 2000, A\&A, 358, 125 
Fletcher, A., Korpi, M., \& Shukurov, A. 2009, in IAU Symp., 259, 87 Ghez, A. M., Salim, S., Weinberg, N. N., et al. 2008, ApJ, 689, 1044 Gillessen, S., Eisenhauer, F., Trippe, S., et al. 2009, ApJ, 692, 1075 Goto, M., Usuda, T., Nagata, T., et al. 2008, ApJ, 688, 306 Gray, A. D., Cram, L. E., Ekers, R. D., et al. 1991, Nature, 353, 237 Gray, A. D., Nicholls, J., Ekers, R. D., et al. 1995, ApJ, 448, 164 Han, J. 2002, in Astrophysical Polarized Backgrounds, ed. S. Cecchini, S. Cortiglioni, R. Sault, \& C. Sbarra, AIP Conf. Ser., 609, 96

Han, J. L., Manchester, R. N., Berkhuijsen, E. M., et al. 1997, A\&A, 322, 98

Han, J. L., Manchester, R. N., Lyne, A. G., Qiao, G. J., \& van Straten, W. 2006, ApJ, 642, 868

Han, J. L., Manchester, R. N., \& Qiao, G. J. 1999, MNRAS, 306, 371

Hanasz, M., Kowal, G., Otmianowska-Mazur, K., et al. 2004, ApJ, 605, L33

Hanasz, M., Otmianowska-Mazur, K., Lesch, H., et al. 2009, in IAU Symp., 259, 479

Heyvaerts, J., Norman, C., \& Pudritz, R. E. 1988, ApJ, 330, 718

Hildebrand, R. H. 1988, QJRAS, 29, 327

Inoue, M., \& Tabara, H. 1981, PASJ, 33, 603

Inoue, M., Takahashi, T., Tabara, H., Kato, T., \& Tsuboi, M. 1984, PASJ, 36, 633

Killeen, N. E. B., Lo, K. Y., \& Crutcher, R. 1992, ApJ, 385, 585

Knödlseder, J., Jean, P., Lonjou, V., et al. 2005, A\&A, 441, 513

Koyama, K., Maeda, Y., Sonobe, T., et al. 1996, PASJ, 48, 249

Krause, M. 2009, Rev. Mex. Astron. Astrofis. Ser. Conf., 36, 25

Kulsrud, R. M., Cen, R., Ostriker, J. P., et al. 1997, ApJ, 480, 481

Kulsrud, R. M., \& Zweibel, E. G. 2008, Rep. Progr. Phys., 71, 046901

Lacey, C. G., \& Fall, S. M. 1985, ApJ, 290, 154

Lang, C. C., Anantharamaiah, K. R., Kassim, N. E., et al. 1999a, ApJ, 521, L41

Lang, C. C., Morris, M., \& Echevarria, L. 1999b, ApJ, 526, 727

LaRosa, T. N., Kassim, N. E., Lazio, T. J. W., et al. 2000, AJ, 119, 207

LaRosa, T. N., Nord, M. E., Lazio, T. J. W., et al. 2004, ApJ, 607, 302

LaRosa, T. N., Brogan, C. L., Shore, S. N., et al. 2005, ApJ, 626, L23

Lesch, H., \& Reich, W. 1992, A\&A, 264, 493

Lesch, H., Crusius, A., Schlickeiser, R., et al. 1989, A\&A, 217, 99

Liszt, H. S. 1985, ApJ, 293, L65

Liszt, H. S., \& Burton, W. B. 1980, ApJ, 236, 779

Marshall, J., Lasenby, A. N., \& Yusef-Zadeh, F. 1995, MNRAS, 274, 519

Mayer-Hasselwander, H. A., Bertsch, D. L., Dingus, B. L., et al. 1998, A\&A, 335,161

Men, H., Ferrière, K., \& Han, J. L. 2008, A\&A, 486, 819

Meyer, L., Schödel, R., Eckart, A., et al. 2007, A\&A, 473, 707

Mezger, P. G., Duschl, W. J., \& Zylka, R. 1996, A\&AR, 7, 289

Miley, G. 1980, ARA\&A, 18, 165

Morris, M. 1990, in Galactic and Intergalactic Magnetic Fields, ed. R. Beck, R. Wielebinski, \& P. P. Kronberg, IAU Symp., 140, 361

Morris, M. 1996, in Unsolved Problems of the Milky Way, ed. L. Blitz, \& P. J. Teuben, IAU Symp. 169, 247

Morris, M. 2007, ArXiv Astrophysics e-prints

Morris, M., Davidson, J. A., Werner, M., et al. 1992, ApJ, 399, L63

Morris, M., \& Serabyn, E. 1996, ARA\&A , 34, 645
Morris, M., Uchida, K., \& Do, T. 2006, Nature, 440, 308

Morris, M., \& Yusef-Zadeh, F. 1989, ApJ, 343, 703

Moss, D., \& Sokoloff, D. 2008, A\&A, 487, 197

Muno, M. P., Baganoff, F. K., Bautz, M. W., et al. 2004, ApJ, 613, 326

Nishiyama, S., Tamura, M., Hatano, H., et al. 2009, ApJ, 690, 1648

Nord, M. E., Lazio, T. J. W., Kassim, N. E., et al. 2004, AJ, 128, 1646

Noutsos, A., Johnston, S., Kramer, M., et al. 2008, MNRAS, 386, 1881

Novak, G., Chuss, D. T., Renbarger, T., et al. 2003, ApJ, 583, L83

Novak, G., Dotson, J. L., Dowell, C. D., et al. 2000, ApJ, 529, 241

Ott, T., Eckart, A., \& Genzel, R. 1999, ApJ, 523, 248

Parker, E. N. 1992, ApJ, 401, 137

Plante, R. L., Lo, K. Y., \& Crutcher, R. M. 1995, ApJ, 445, L113

Prouza, M., \& Šmída, R. 2003, A\&A, 410, 1

Rand, R. J., \& Kulkarni, S. R. 1989, ApJ, 343, 760

Rand, R. J., \& Lyne, A. G. 1994, MNRAS, 268, 497

Reich, W., Wielebinski, R., Sofue, Y., et al. 1988, A\&A, 191, 303

Roy, S., Pramesh Rao, A., \& Subrahmanyan, R. 2008, A\&A, 478, 435

Roy, S., Rao, A. P., \& Subrahmanyan, R. 2005, MNRAS, 360, 1305

Sawada, T., Hasegawa, T., Handa, T., et al. 2004, MNRAS, 349, 1167

Schwarz, U. J., \& Lasenby, J. 1990, in Galactic and Intergalactic Magnetic

Fields, ed. R. Beck, R. Wielebinski, \& P. P. Kronberg, IAU Symp., 140, 383

Shore, S. N., \& Larosa, T. N. 1999, ApJ, 521, 587

Sofue, Y. 1984, PASJ, 36, 539

Sofue, Y., \& Fujimoto, M. 1987, PASJ, 39, 843

Sofue, Y., \& Handa, T. 1984, Nature, 310, 568

Sun, X. H., Reich, W., Waelkens, A., et al. 2008, A\&A, 477, 573

Trippe, S., Paumard, T., Ott, T., et al. 2007, MNRAS, 375, 764

Tsuboi, M., Inoue, M., Handa, T., Tabara, H., \& Kato, T. 1985, PASJ, 37, 359

Tsuboi, M., Inoue, M., Handa, T., et al. 1986, AJ, 92, 818

Tsuboi, M., Kawabata, T., Kasuga, T., Handa, T., \& Kato, T. 1995, PASJ, 47, 829

Uchida, K. I., \& Guesten, R. 1995, A\&A, 298, 473

Vallée, J. P. 2005, ApJ, 619, 297

Weidenspointner, G., Shrader, C. R., Knödlseder, J., et al. 2006, A\&A, 450, 1013

Yamauchi, S., Kawada, M., Koyama, K., Kunieda, H., \& Tawara, Y. 1990, ApJ, 365,532

Yusef-Zadeh, F. 2003, ApJ, 598, 325

Yusef-Zadeh, F., Hewitt, J. W., \& Cotton, W. 2004, ApJS, 155, 421

Yusef-Zadeh, F., \& Morris, M. 1987a, AJ, 94, 1178

Yusef-Zadeh, F., \& Morris, M. 1987b, ApJ, 322, 721

Yusef-Zadeh, F., \& Morris, M. 1988, ApJ, 329, 729

Yusef-Zadeh, F., Morris, M., \& Chance, D. 1984, Nature, 310, 557

Yusef-Zadeh, F., Roberts, D. A., Goss, W. M., Frail, D. A., \& Green, A. J. 1996, ApJ, 466, L25

Yusef-Zadeh, F., Roberts, D. A., Goss, W. M., Frail, D. A., \& Green, A. J. 1999, ApJ, 512, 230

Yusef-Zadeh, F., Wardle, M., Muno, M., Law, C., \& Pound, M. 2005, Adv. Space Res., 35, 1074

Yusef-Zadeh, F., Wardle, M., \& Parastaran, P. 1997, ApJ, 475, L119 